\title{
Carbon stars and C/M ratio in the WLM dwarf irregular galaxy ${ }^{\star}$
}

\author{
A. T. Valcheva ${ }^{1}$, V. D. Ivanov ${ }^{2}$, E. P. Ovcharov ${ }^{1}$, and P. L. Nedialkov ${ }^{1}$ \\ 1 Department of Astronomy, University of Sofia, 5 James Bourchier, Sofia 1164, Bulgaria \\ e-mail: [valcheva; evgeni; japet] @phys.uni-sofia.bg \\ 2 European Southern Observatory, Ave. Alonso de Cordova 3107, Casilla 19, Santiago 19001, Chile \\ e-mail: vivanov@eso.org
}

Received 17 November 2005 / Accepted 18 January 2007

ABSTRACT

\begin{abstract}
We identify the rich carbon star population of the Magellanic-type dwarf irregular galaxy WLM (Wolf-Lundmark-Melotte) and study its photometric properties from deep near-IR observations. The galaxy also exhibits a clear presence of oxygen-rich population. We derive a carbon to M-star ratio of $C / M=0.56 \pm 0.12$, relatively high in comparison with many galaxies. The spatial distribution of the AGB stars in WLM hints at the presence of two stellar complexes with a size of a few hundred parsecs. Using the H I map of

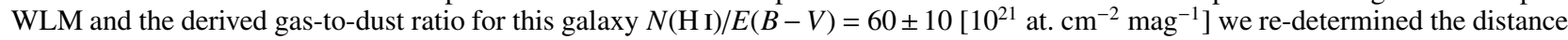
modulus of WLM from the IR photometry of four known Cepheids, obtaining $(m-M)_{0}=24.84 \pm 0.14 \mathrm{mag}$. In addition, we determine the scale length of $0.75 \pm 0.14 \mathrm{kpc}$ of WLM disk in the $J$-band.
\end{abstract}

Key words. galaxies: individual: WLM - stars: AGB and post-AGB - stars: carbon - galaxies: irregular - Local Group

\section{Introduction}

WLM (Wolf-Lundmark-Melotte; also DDO 221; Wolf 1909; Melotte 1926) is a dwarf irregular Local Group member. Earlier photographic surveys of the galaxy were presented in Ables \& Ables (1977) and Sandage \& Carlson (1985). The first CCD observations of WLM were performed by Ferraro et al. (1989), who reported significant variations in the recent star-formation rate across the galaxy. They also detected a uniform underlying relatively old stellar population $(\sim 1 \mathrm{Gyr})$. Later on, Minniti \& Zijlstra (1997) derived a distance modulus $(m-M)_{0}=$ $24.75 \pm 0.1 \mathrm{mag}$ and $[\mathrm{Fe} / \mathrm{H}]=-1.45 \pm 0.2$ from $V$ - and $I$-band photometry. Hodge et al. (1999) reported the first HST observations of WLM. They resolved the sole globular cluster (Ables $\&$ Ables 1977) and obtained $(m-M)_{0}=24.73 \pm 0.07 \mathrm{mag}$, $[\mathrm{Fe} / \mathrm{H}]=-1.52 \pm 0.08$, and an age of $14.8 \pm 0.6 \mathrm{Gyr}$, typical for the old globulars in the Milky Way. Dolphin (2000) concluded that the WLM started to form stars about 12 Gyr ago, with approximately half of the star-formation occurring during the last 9 Gyr, also based on HST imaging.

Recently, Venn et al. (2003) determined $[\mathrm{Fe} / \mathrm{H}]=-0.38 \pm$ 0.29 from high-resolution spectroscopy of two WLM blue supergiants. They found depleted stellar oxygen abundance by a factor of five, in comparison with the oxygen abundance of the HII regions. Even though later Lee et al. (2005) reduced the discrepancy, some unusual chemical evolution history is required to explain the observations because the enhanced stellar abundance would put WLM well above the metallicity-luminosity relation.

These results make it important to study the carbon stars in WLM because of their sensitivity to the metal abundance. Besides, AGB stars are representative of the stellar population with ages between 1-10 Gyr and can be used as an observational constraint to the properties of the post-main sequence stellar

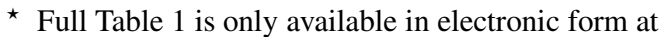
http://www. aanda.org evolution. Initially, the behavior of the $\mathrm{C} / \mathrm{M}$ ratio is understood at least qualitatively (Scalo \& Miller 1981; Iben \& Renzini 1983). A large number of studies of AGB stars in Local Group galaxies were performed using optical narrow-band imaging. It turns out to be an easy way to separate M-type from carbon stars (Albert et al. 2000; Battinelli \& Demers 2004). The progress in recent years made it possible to produce the first observational calibrations of the $\mathrm{C} / \mathrm{M}$ ratio versus metallicity relation (Groenewegen 2006; Cioni \& Habing 2005; Battinelli \& Demers 2005).

As AGB stars are almost the brightest cool stars it is easy to investigate them in the near-IR range. The interest in this region of the spectrum grew strongly in the last three years (Cioni \& Habing 2003, 2005; Kang et al. 2005, 2006; Sohn et al. 2006). Here we report on the analysis of the carbon stars in WLM from near-IR imaging.

\section{Observations and data reduction}

The observations of WLM were made in Dec. 2004 under nonphotometric conditions. To estimate the foreground contamination, an additional field located 14.8 arcmin South from the center of the galaxy, far enough to eliminate the galaxy contribution, was observed in July 2006 (hereafter "clear sky" field). The near-IR imaged and spectrograph SofI (Son of ISAAC) at the ESO NTT (New Technology Telescope) on La Silla was used. The instrument is equipped with a $1024 \times 1024$ Hawaii $\mathrm{HgCdTe}$ array with 18.5 micron square pixels and a scale of 0.288 arcsec pixel $^{-1}$, giving a field of view of $4.92 \times$ $4.92 \mathrm{arcmin}^{2}$. We alternated between the target and a clear patch of sky nearby. To minimize the effects of array cosmetics and to improve the sky subtraction, we introduced a small random jitter of up to a few tens of arcseconds between the sequential images. In total, we collected 10 images for both fields in $J_{\mathrm{S}}$ and 20 on the galaxy and 15 on the "clear sky" field in $K_{S}$. Each of the images in $J_{\mathrm{S}}$ is the average of $2 \times 30 \mathrm{~s}$ exposures and of 


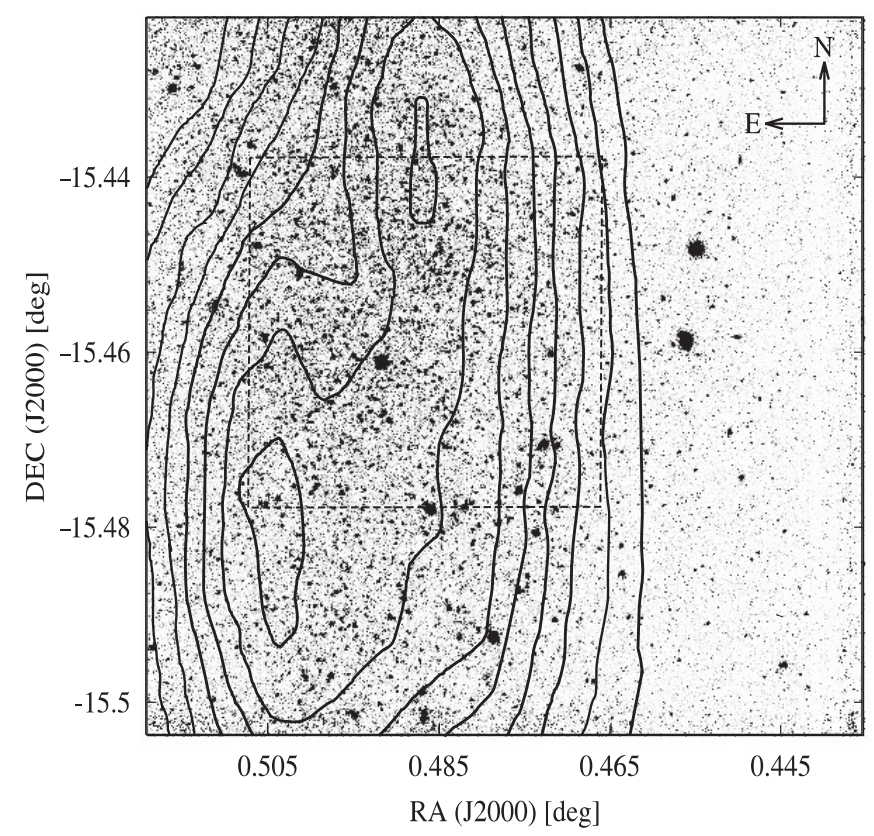

Fig. 1. Near-IR image of WLM. Isolines show the H I map of Jackson et al. (2004) at levels from 25 to $95 \%$ of the maximum column density $\left(2.16 \times 10^{21} \mathrm{~cm}^{-2}\right)$, in increments of $10 \%$. The square marks the boundary of the "inner" field (see Sect. 2).

$8 \times 8 \mathrm{~s}$ exposures in $K_{\mathrm{S}}$. The total integration time was $10 \mathrm{~min}$ in $J_{\mathrm{S}}$ (hereafter $J$ for simplicity) and, in $K_{\mathrm{S}}, 21.33 \mathrm{~min}$ on the galaxy and 16 min on the "clear sky".

The data reduction followed the typical steps. First, we created a sky for each filter by median combination of the off-target images using suitable upper limits and rejection algorithms to exclude the stars present in the sky field. Next, we subtracted the sky from each image, and divided it by the dome flat. The dome flats are preferable in comparison to the sky flats because they allow us to remove the variable bias level, found in some Hawaii detectors. To account for the uneven illumination of the dome flat screen, we applied an illumination correction. It was derived from the photometry of a standard star, placed on 16 positions across the field of average view, in a $4 \times 4$ grid. Finally, the individual images were aligned and combined to form the final image (Fig. 1).

The photometric calibration was performed by comparing our instrumental photometry with the measurements from the 2MASS Point Source Catalog:

$$
\begin{aligned}
& J=j-5.55( \pm 0.05) \\
& K_{\mathrm{S}}=k-8.65( \pm 0.18)
\end{aligned}
$$

based on 18 common stars. Here $J$ and $K_{\mathrm{S}}$ are the 2MASS systematic magnitudes, and $j$ and $k$ are the instrumental magnitudes. No statistically significant color terms were found.

The photometry was performed with the IRAF DAOPHOT package. In the galaxy field, 1550 stars were detected in $J$ and 739 in $K_{\mathrm{S}}$ with a threshold of $4 \sigma$ and $3 \sigma$, respectively, above the background. The instrumental magnitudes for each filter were obtained by using constant Gaussian PSF. The positions and magnitudes of all measured stars matched in both $J$ and $K_{\mathrm{S}}$ frames with a maximum tolerance of 3 pixels $(0.86 \operatorname{arcsec})$ are given in Table 1. The columns contain: identification number, RA and DEC (J2000), $J$ and $K_{\mathrm{S}}$ magnitudes (the $1 \sigma$ errors are

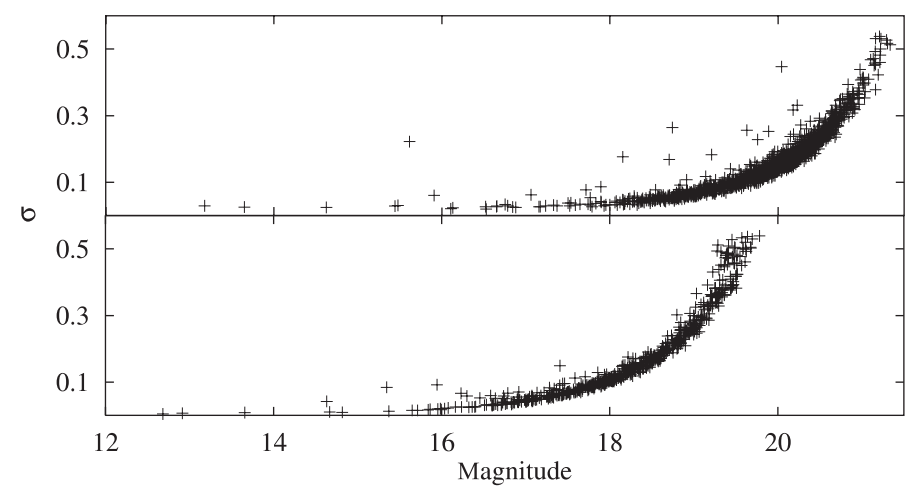

Fig. 2. Photometric errors as a function of the magnitude for the $J$ - (top) and $K_{\mathrm{S}^{-}}$(bottom) bands. The completeness limits of our photometry are $\sim 20.1$ and $\sim 17.9$ mag for the $J$ - and $K_{\mathrm{S}}$-bands, respectively.

Table 1. Photometry of $555 \mathrm{WLM}$ stars with $J$ - and $K_{\mathrm{S}}$-band photometry.

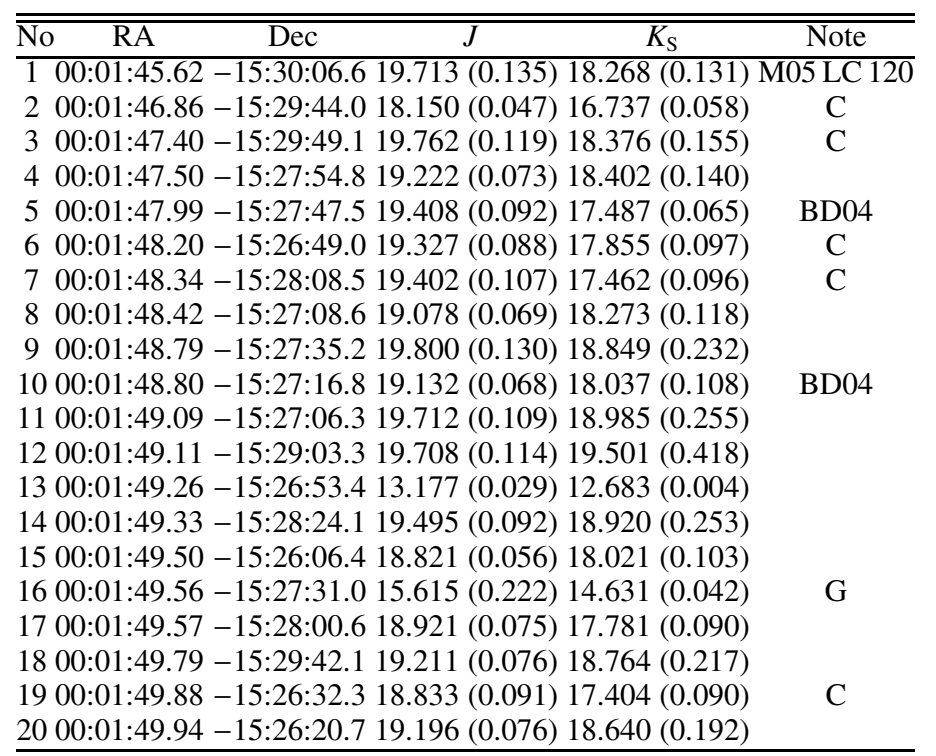

The full table is available in the electronic edition.

given in brackets (keys to the notes: V - variable star, with identifier from the source paper (SC85 for Sandage \& Carlson 1985 and M05 for Mora et al. in preparation), C - carbon star candidate (BD04 for Battinelli \& Demers 2004), G - globular cluster). The paper edition of the journal contains only the first 20 entries. The rest of the table is available in the electronic version of the journal only. The measurement errors are plotted in Fig. 2.

The reduction and calibration procedures for the "clear sky" field follow analogous steps. The photometric equations obtained from 7 2MASS stars

$J=j-3.12( \pm 0.03)$

$K_{\mathrm{S}}=k-5.34( \pm 0.04)$

were used to transform the magnitudes of 89 stars in $J$ and 67 in $K_{\mathrm{S}}$ into $2 \mathrm{MASS}$ magnitudes. The matching procedure gives 50 common stars in $J$ and $K_{\mathrm{S}}$ in total.

The luminosity functions in $J$ and $K_{\mathrm{S}}$ are shown in Fig. 3. The number of stars in each bin is normalized to 1 square arcmin. We tested the completeness limit variations across the galaxy by splitting the field into an "inner" part, containing the area with maximum H I column densities, and an "outer" part, respectively 

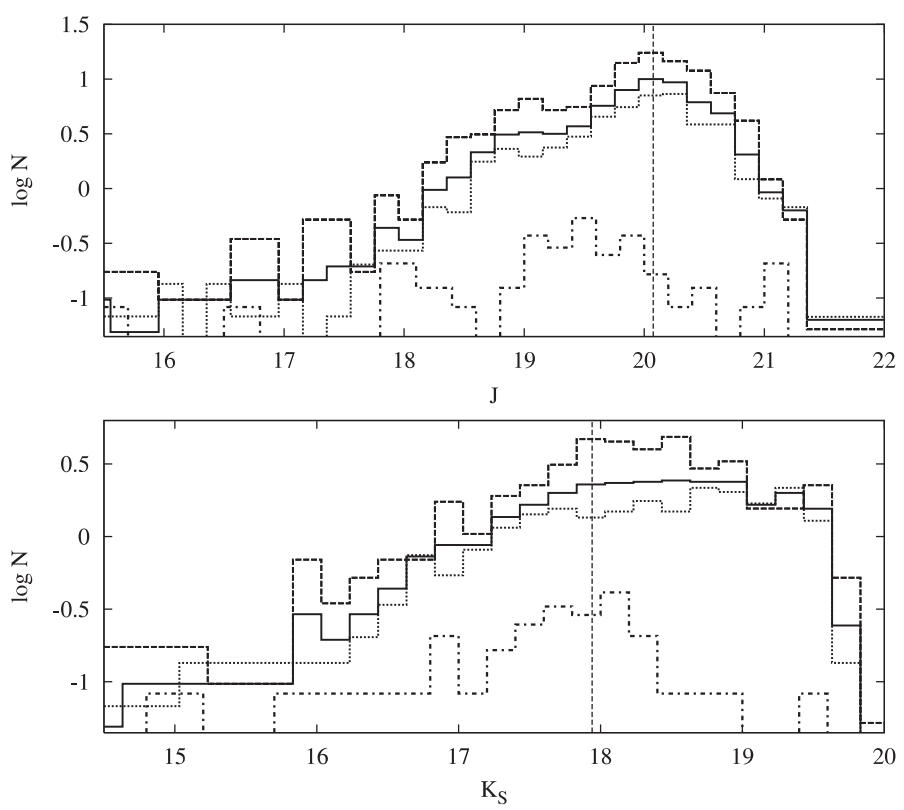

Fig. 3. Luminosity functions for $J$ (upper panel) and $K_{\mathrm{S}}$ (bottom panel) with a magnitude bin of $0.2 \mathrm{mag}$. $\log N$ is the number of stars normalized for 1 square arcmin. Each panel shows the luminosity functions for the whole field (solid lines), the inner part (dashed lines), and outer part (dotted lines). The LF for the "clear sky" field is also shown with dot-dashed lines. Vertical lines represent our galaxy 100\% photometry completeness for every band.

5.76 and 14.77 square arcmin (see Fig. 1). The crowding effects are limited to $\leq 0.2 \mathrm{mag}$. We adopted $J^{\mathrm{lim}} \sim 20.1 \mathrm{mag}$ and $K_{\mathrm{S}}^{\mathrm{lim}} \sim 17.9 \mathrm{mag}$. For comparison the luminosity function of the "clear sky" is also shown in Fig. 3. On average the LF is nearly an order lower then the galaxy one.

\section{Color-magnitude diagram}

The color-magnitude diagram of WLM is shown in Fig. 4 (top panel). We used distance modulus $(m-M)_{0}=24.85 \mathrm{mag}-$ an average of the estimates of: (i) Minniti \& Zijlstra (1997), who derived $(m-M)_{0}=24.75 \pm 0.1 \mathrm{mag}$ from the $I$-magnitude of the red giant branch (hereafter RGB) tip, and (ii) Rejkuba et al. (2000), who obtained $(m-M)_{0}=24.95 \pm 0.13$ mag from $V$-band photometry of the horizontal branch. These two values agree within the errors and they are derived from different methods and data sets. Corrections for the Galactic extinction were made using $E(B-V)=0.037 \mathrm{mag}$ (Schlegel et al. 1998). Here and throughout the paper we use the extinction law of Rieke \& Lebofsky (1985).

For the high Galactic latitude of WLM $\left(b=-73.62^{\circ}\right)$, we expect the foreground contamination to be negligible. This is confirmed from the number of stars found under similar observational conditions in our "clear sky", which is just $9 \%$ of all stars in the galaxy field. The color-magnitude diagram was constructed using the same distance modulus and Galactic extinction and is shown in Fig. 4 (right panel). In such way a true estimation of the Galaxy contamination can be made by counting the stars in 1 magnitude bins in $M_{K_{\mathrm{S}}}$. The numbers are given on the right in Fig. 4 (left panel).

The comparison with isochrones of Bertelli et al. (1994) indicates that the majority of the stars are 1-10 Gyr old, with a significant population of AGB and carbon stars with $\left(J-K_{\mathrm{S}}\right)_{0}>$ $1 \mathrm{mag}$. Only a handful of blue stars with $\left(J-K_{\mathrm{S}}\right)_{0}<1 \mathrm{mag}$ and $M_{K_{\mathrm{S}}}>-8 \mathrm{mag}$ are detected. We also identified a number of variable stars on our images from Sandage \& Carlson (1985) and Mora et al. (in preparation). The RGB properties versus metallicity calibrations of Ivanov \& Borissova (2002) predict that the RGB tip for the WLM metallicity is located at $M_{K_{\mathrm{S}}}>$ $-6.14 \mathrm{mag}$. Unfortunately, the incompleteness of the photometry in this magnitude range prevents us from analysis of the RGB properties.

\section{Carbon star identification and $\mathrm{C} / \mathrm{M}$ ratio in the WLM}

WLM is an unusually rich carbon star, along with two other dwarf galaxies - IC 1613 and NGC 6822 - according to Cook et al. (1986). Recently, Battinelli \& Demers (2004) surveyed WLM using narrow band $\mathrm{CN}-\mathrm{TiO}$ filters and identified 149 carbon stars and practically no M-type AGB stars (77 identified carbon stars in our field are marked in Table 1 with BD04). They also found an extremely high C/M ratio of WLM: $12.4 \pm 3.7$.

The IR photometry allows us to carry out an independent census of the carbon stars, following the method applied on the Magellanic Clouds (Cioni \& Habing 2003), NGC 6822 (Cioni \& Habing 2005, Kang et al. 2006), NGC 185 (Kang et al. 2005), and NGC 147 (Sohn et al. 2006). It is found that the color distribution of all AGB stars have a well-pronounced discontinuity at some point, M-type stars have a peak followed by a red tail of carbon stars. We separated the carbon-rich from the oxygen-rich stars using the color distribution of the stars above the RGB tip (Fig. 6, the inset) and found color limit at $\left(J-K_{\mathrm{S}}\right)_{0}=1.20 \mathrm{mag}$. A similar dereddened limit was found by Cioni \& Habing (2005) for NGC 6822, and Sohn et al. (2006) for NGC 147: $\left(J-K_{\mathrm{S}}\right)_{0}=$ 1.24 and $\left(J-K_{\mathrm{S}}\right)_{0}=1.28$, respectively.

Integrating over the two parts of the histogram we obtain 146 carbon stars and $259 \mathrm{M}$ stars. The latter number includes all stars with spectral types M0 and later (M0, M1, M2, etc.), which is sometimes designated as $\mathrm{M} 0+$. Using the same criteria on the "clear sky" field we obtain $31 \mathrm{C}$ stars and $19 \mathrm{M}$ stars as all of them are above the RGB tip. Taking into account the latter numbers, this will reduce our $\mathrm{C}$ stars to 115 and the $\mathrm{M}$ star to 240 .

The $K_{\mathrm{S}}$ luminosity functions for our carbon star sample and for the 77 cross-identified carbon stars (shown in Fig. 6) from Battinelli \& Demers (2004) are shown in Fig. 5. To eliminate the binning effects, we created two luminosity functions for each data set, shifting the bin centers by half of the bin width, and averaged them. We fitted the luminosity function with a Gaussian, in two iterations, excluding the values that deviated more than $3 \sigma$. The final fits have a mean of $\left\langle K_{\mathrm{S}}\right\rangle=17.35 \mathrm{mag}, \sigma=$ $0.71 \mathrm{mag}$ for our sample and $\left\langle K_{\mathrm{S}}\right\rangle=17.33 \mathrm{mag}, \sigma=0.51 \mathrm{mag}$ for the 77 carbon stars from Battinelli \& Demers (2004), which correspond to $\left\langle M_{K_{\mathrm{S}}}\right\rangle=-7.51 \mathrm{mag}$ and $\left\langle M_{K_{\mathrm{S}}}\right\rangle=-7.53 \mathrm{mag}$, respectively.

Previous studies in some LG galaxies in the near IR lead to identical mean absolute $K$ magnitude of the $\mathrm{C}$ stars: $\left\langle M_{K_{\mathrm{S}}}\right\rangle=$ $-7.93 \pm 0.36 \mathrm{mag}$ (Kang et al. 2005), $\left\langle M_{K_{\mathrm{S}}}\right\rangle=-7.56 \pm 0.47 \mathrm{mag}$ (Sohn et al. 2006), and $\left\langle M_{K_{\mathrm{S}}}\right\rangle=-7.60 \pm 0.50 \mathrm{mag}$ (Kang et al. 2006) for NGC 185, NGC 147 and NGC 6822, respectively. $\left\langle M_{K_{\mathrm{S}}}\right\rangle$ in WLM is comparable with these values in $1 \sigma$ level and support this assumption. The sample of galaxies includes dwarf irregulars and dwarf ellipticals, but is not representative of a wide range of metallicity and a possible influence over the mean absolute $K$ magnitude cannot be entirely excluded.

Next, we derived a $\mathrm{C} / \mathrm{M}$ ratio of $0.56 \pm 0.12$ (foreground corrected ratio 0.48 ), using the carbon and M-type stars we 


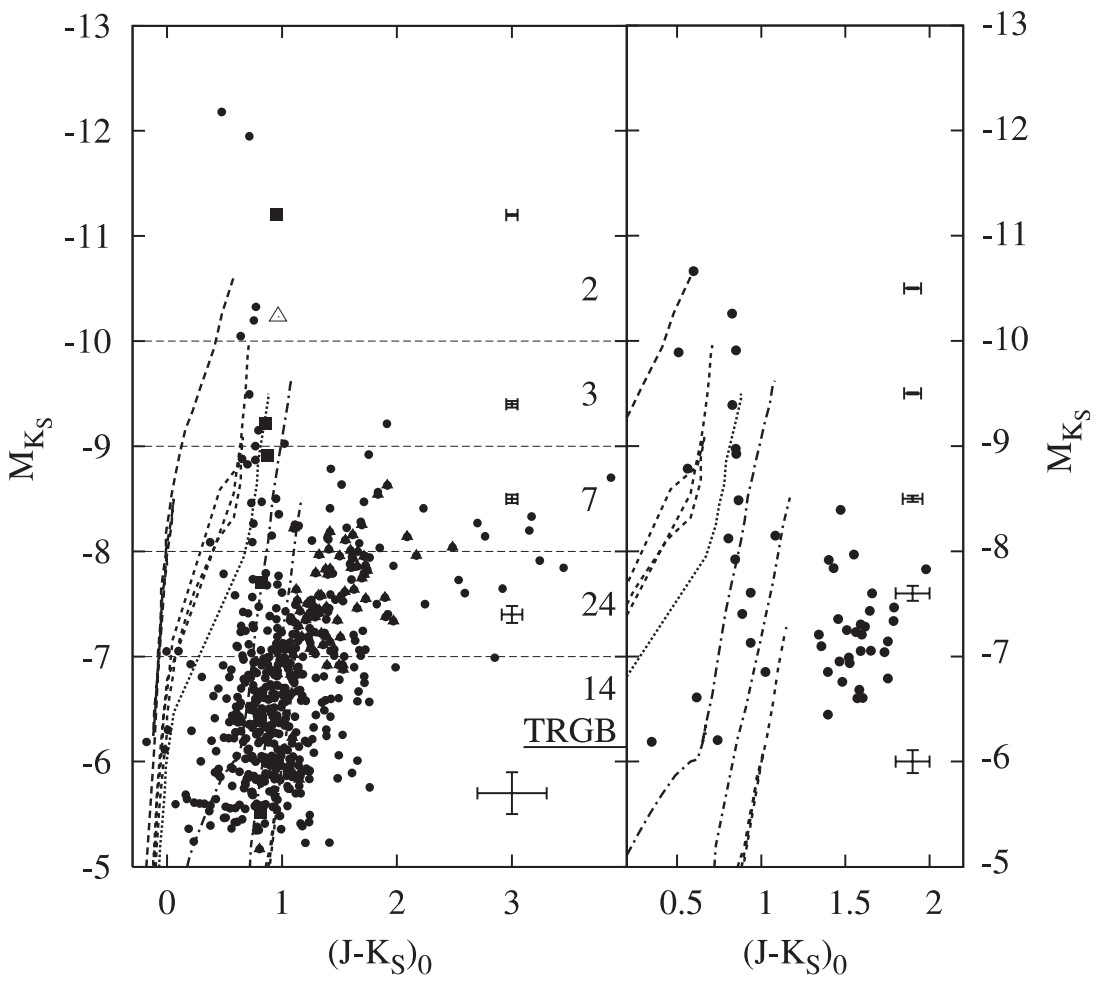

Fig. 4. Near-IR $M_{K_{\mathrm{S}}}$ versus $\left(J-K_{\mathrm{S}}\right)_{0}$ mag colormagnitude diagram of WLM (left panel) and the "clear sky" field (right panel; see Sect. 3 for details). The typical measurement errors for stars with $\left(J-K_{\mathrm{S}}\right)_{0}=$ 1 mag are shown on the right. Isochrones for the 10,20 , 30, $100 \mathrm{Myr}, 1$ and $10 \mathrm{Gyr}$, and $\mathrm{Z}=0.001$ from Bertelli et al. (1994) are plotted. Left panel: the open triangle indicates the location of the WLM globular cluster. Six variable stars from Sandage \& Carlson (1985) are plotted with solid squares and 77 carbon stars of Battinelli \& Demers (2004) are shown with solid triangles. The level of the RGB tip is also marked on the right. The number of foreground stars in every 1-mag bin along $M_{K_{\mathrm{S}}}$ obtained from CMD of "clear sky" are given on the right.

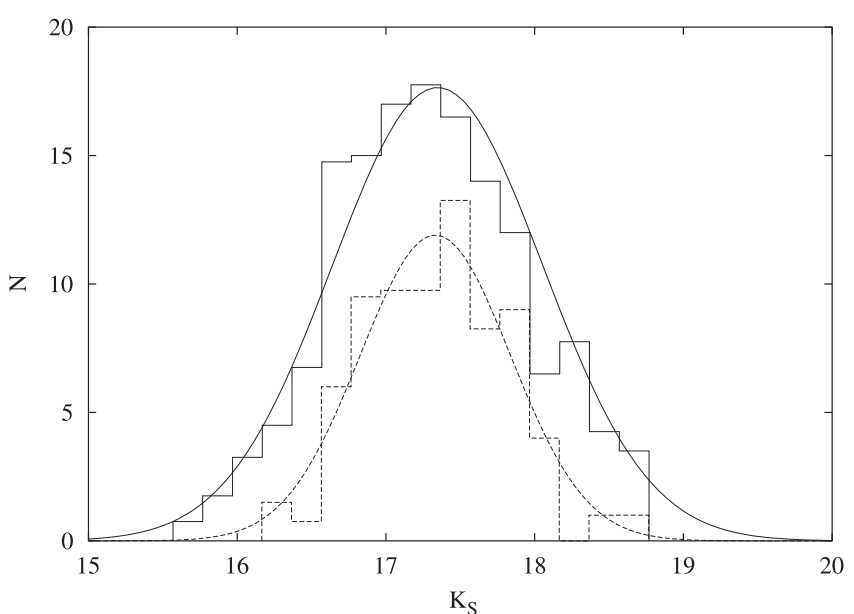

Fig. 5. Luminosity functions of the carbon stars in WLM. The solid line represents the distribution of carbon stars above the RGB tip. The dashed line shows the 77 carbon stars from Battinelli \& Demers (2004) that we identified in our field. The bin width is $0.2 \mathrm{mag}$. The final Gaussian fits to the data are shown (see Sect. 4 for details).

identified above the RGB tip. However, Cioni \& Habing (2005) pointed that this classification method omits the early-type carbon stars that might be bluer than the adopted color limit. The incompleteness due to this effect can be estimated by comparison of our carbon star list and that of Battinelli \& Demers (2004) it yielded additional 15 carbon stars among the $\mathrm{M}$ stars, bringing the $\mathrm{C} / \mathrm{M}$ ratio up to $0.66 \pm 0.11(0.58)$. The difference between the two values is small, considering the Poisson uncertainties given here.

Both values obtained here are smaller by a factor of 20 than the result of Battinelli \& Demers (2004): C/M0+ 12.4. The large difference is due to the presence of the M-type AGB population, which is almost entirely absent in Battinelli \& Demers' (2004) survey of WLM. We can only speculate that this difference is related to the narrow-band filter centered at the $\mathrm{TiO}$ absorption feature - prominent for spectral types later than M3. Their method probably omits early M-type stars. Furthermore, the low metallicity of WLM additionally diminishes the TiO feature strength and makes the detection of M-type stars more difficult.

\section{Spatial distribution of the stellar populations}

Our data cover most of the main body of WLM, allowing us to study the spatial distribution of stellar populations. We created 2-dimensional histograms (Fig. 7) with $22.5 \times 22.5 \mathrm{arcsec}^{2}$ bins, corresponding to a linear scale of $100 \times 100 \mathrm{pc}$ at the distance of the galaxy. The distributions were smoothed by a box-car function of width 2.

The histograms indicate no major difference between the stellar distributions. There are two peaks, corresponding to complexes with typical sizes of a few hundred parsecs. The C/M ratio exhibits a more complicated behavior - the peak is slightly offset from the peak of the stellar distributions. Further studies are necessary to verify if this reflects a real metallicity or an age gradient across the galaxy.

\section{Miscellaneous}

\subsection{Cepheid distance to WLM in the near-IR}

We identified 5 Cepheids from the list of Sandage \& Carlson (1985) on our $J$-band image (the $K_{\mathrm{S}}$ image is too shallow to detect them). We derived a distance to WLM adopting the periodluminosity relation of Gieren et al. (1998):

$M_{J}=-5.240( \pm 0.028)-3.129( \pm 0.052) \times(\log P-1.0)$,

where $M_{J}$ is the absolute $J$-band magnitude of the Cepheids and $P$ is the period in days. The distances to individual Cepheids are listed in Table 2. The columns contain: identification number, 


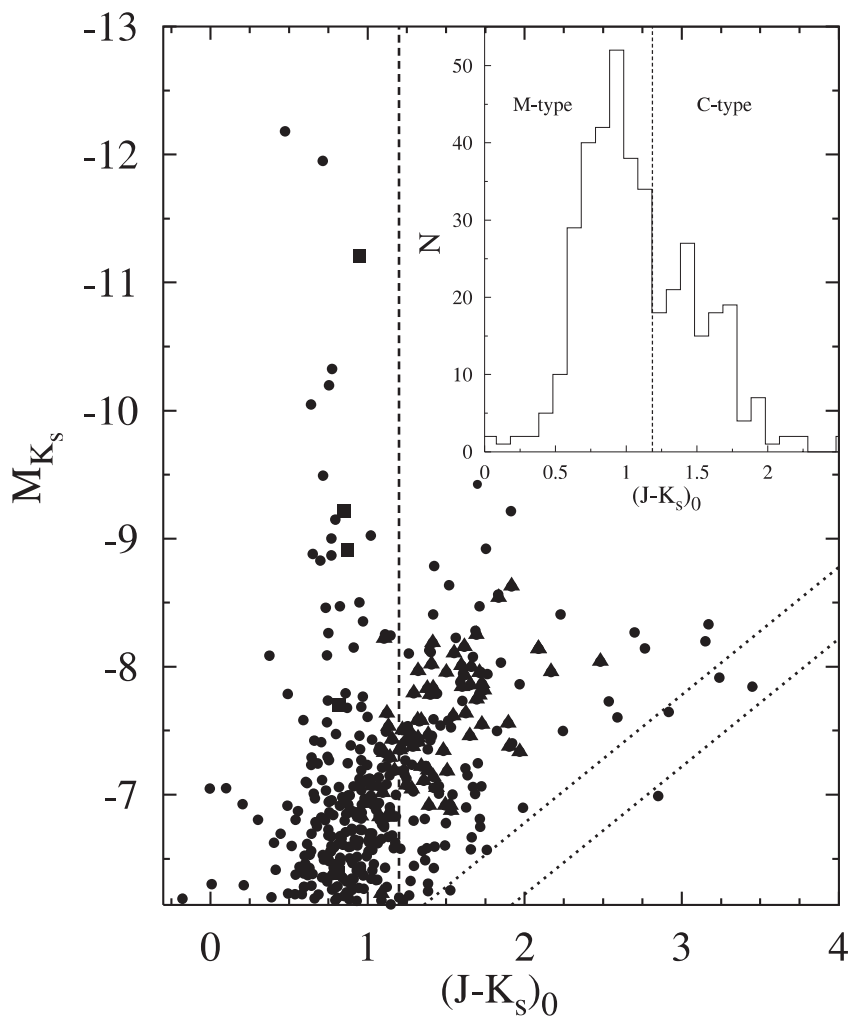

Fig. 6. Color-magnitude $M_{K_{\mathrm{S}}}$ versus $\left(J-K_{\mathrm{S}}\right)_{0}$ diagram for AGB stars in WLM. The two dotted lines represent the $100 \%$ and $25 \%$ photometry completeness limits. Six variables from Sandage \& Carlson (1985) are plotted with solid squares and 77 carbon stars from Battinelli \& Demers (2004) are plotted with solid triangles. The inset is a color distribution of the stars above the RGB tip, used to separate the oxygen-rich and carbon-rich stars. See Sect. 4 for details. The separation line (dashed line) $\left(J-K_{\mathrm{S}}\right)_{0}=1.20$ is shown on both panels.

$J$-band magnitude (the $1 \sigma$ errors are given in brackets), period in days from Sandage \& Carlson (1985), absolute magnitude calculates from Eq. (5), apparent distance modulus, total extinction in $A_{J}$ derived from gas-to-dust ratio (including Milky Way extinction of $A_{J}=0.033 \mathrm{mag}$, Schlegel et al. 1998), and true distance modulus $(m-M)_{0}$. We removed the Milky Way extinction using $A_{J}=0.033 \mathrm{mag}$. To correct for the internal extinction towards individual Cepheids we used the H I map of Jackson et al. (2004). The hydrogen surface densities were converted into extinction, assuming the gas-to-dust ratio $N(\mathrm{H} \mathrm{I}) / E(B-V)=$ $60 \pm 10.0\left[10^{21}\right.$ at. $\left.\mathrm{cm}^{-2} \mathrm{mag}^{-1}\right]$. This value is an extrapolation based on a linear least-squares fit (Fig. 8) of the gas-to-dust ratio versus $\log (\mathrm{O} / \mathrm{H})+12$ :

$N(\mathrm{H} \mathrm{I}) / E(B-V)=-(58 \pm 5) \times[\log (\mathrm{O} / \mathrm{H})+12]+(517 \pm 44),(6)$

with $\mathrm{rms}=2$.

This relation is based on gas-to-dust ratio estimates for: the solar neighborhood (Bohlin et al. 1978; marked MW in Fig. 8), the M 31 outskirts (Cuillandre et al. 2001; M 31 ), LMC (Junkkarinen et al. 2004; LMC), and SMC (Jakobsson et al. 2004; SMC). The abundances are taken from Russell \& Dopita (1990) for LMC and SMC. In the case of the Milky Way we used the Galactic metallicity gradient from Esteban et al. (2005), and for M 31 - the metallicity gradient of Smartt et al. (2001). Note that this is a preliminary calibration, based on a limited number of measurements. More gas-to-dust ratios are necessary to derive a reliable relation. Finally, we assumed that the Cepheids are located on the WLM mid-plane and therefore, the extinctions
Table 2. Cepheid distance to WLM.

\begin{tabular}{|c|c|c|c|c|c|}
\hline$\overline{\mathrm{No}}$ & Period, days & $M_{J}$ & $\overline{(m-M)_{J}}$ & $A_{J}$ & $(m-1)$ \\
\hline$\overline{55)}$ & 7.6712 & -4.880 & 24.988 & 0.048 & 24.940 \\
\hline 82 & & -4.8 & & $0 .($ & 24.796 \\
\hline 211 & 6 & -5.1 & 75 & 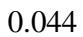 & 31 \\
\hline 481 & 00 & -5.024 & 24.748 & 0.048 & 24.700 \\
\hline $6620.197(0.189)$ & 4.54714 & -4.170 & 24.367 & 0.040 & 24.327 \\
\hline
\end{tabular}

are due to only half of the total hydrogen column densities. The extinction intrinsic to WLM does not exceed $0.015 \mathrm{mag}$, due to the high gas-to-dust ratio of the galaxy.

Rejecting the outlier No. 66 (see Table 2), we obtain a median distance modulus of $(m-M)_{0}=24.84 \pm 0.14$ mag. We tentatively assume that the error is given by the maximum deviation. In addition, there are systematic errors of $0.03 \mathrm{mag}$ for the zero-point of the period-luminosity relation (Eq. (5)), and 0.02 mag for the LMC distance adopted in Gieren et al. (1998).

\subsection{Variable stars in WLM}

Some variable stars from the lists of Sandage \& Carlson (1985) and Mora et al. (in preparation) were included in our field. They are marked in Table 1.

\subsection{Structural parameters of WLM}

Structural parameters of galaxies are determined more reliably from IR images rather than optical ones because the effects of the intrinsic extinction are diminished. Our images do not encompass the entire galaxy, but nevertheless we are able to approximately determine the scale length along the major axis. To do that we averaged the pixel values in a 63.4-arcsec wide strip ( $\sim 300 \mathrm{pc}$ at the distance of WLM), crossing the center of the galaxy in a North-South direction. We used the $J$-band image because of the higher signal-to-noise ratio. The average flux values along the semi-major axis are shown in Fig. 9. An exponential law fit over the central $1 \mathrm{kpc}$ region of WLM yielded a scale length of $0.75 \pm 0.14 \mathrm{kpc}$, similar to the scale length of $0.9 \mathrm{kpc}$ determined by Fisher \& Tully (1975). The inset in Fig. 9 shows histograms of the scale lengths for dwarf galaxies in the Local Group from the compilation of Mateo (1998).

The bright - and younger - stars in the galaxy are resolved on our images, but the fainter - and older ones - are not. We addressed the question if there is a different scale length for different stellar populations by excluding the bright stars from this analysis: we averaged the $J$-band image with a $61 \times 61$ pixel median filter, effectively removing the resolved stars. The derived scale length for the "older" population was $0.71 \pm 0.04 \mathrm{kpc}$, again for the central $1 \mathrm{kpc}$ of the galaxy. Our estimates have to be treated with caution because the strip is not perfectly aligned along the major axis.

\section{Discussion and summary}

This work adds another object to the list of galaxies with homogeneously determined $\mathrm{C} / \mathrm{M} 0+$ ratios from near-IR photometry: LMC, SMC, NGC 6822, NGC 147, NGC 185 and now the WLM. This is an opportunity for an independent check of the results from narrow-band carbon star surveys. The number of data points is still limited and did not allow us to test if using only $\mathrm{C} / \mathrm{M}$ ratios derived from IR observations can reduce the spread of the relation but this is the objective of our further studies. 

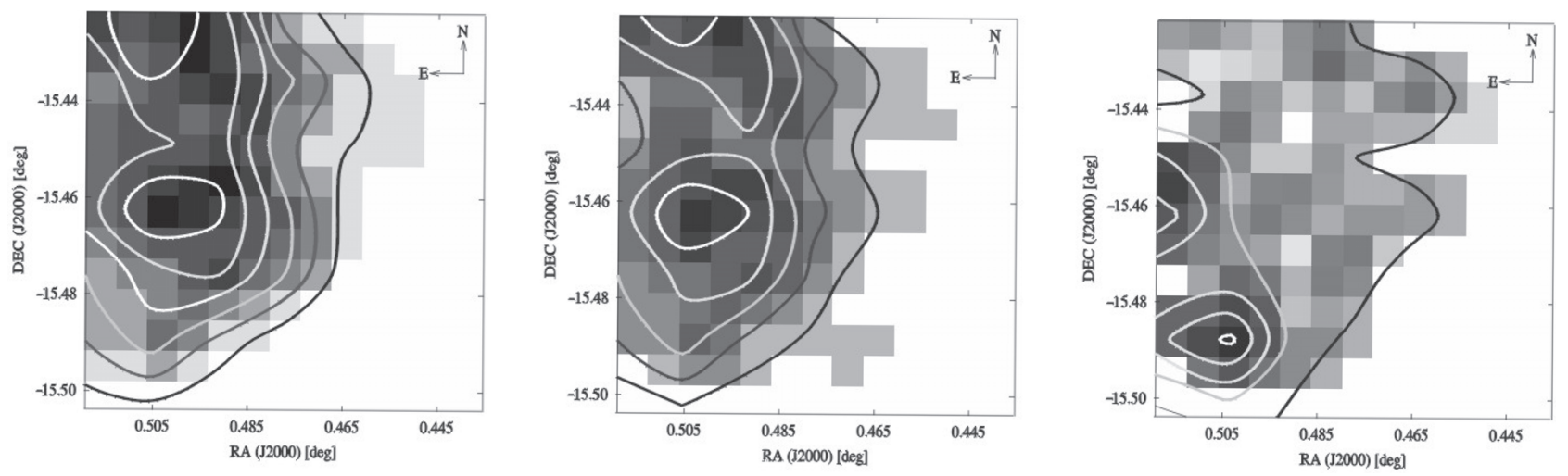

Fig. 7. Spatial distribution of all stars (left), AGB stars (middle), and C/M ratio (right panel). The isolines are at levels: 1 to 6 with a step of 1,1 to 5 with a step of 1 , and 0.2 to 1.4 with a step of 0.3 , respectively, for the three plots from left to right.

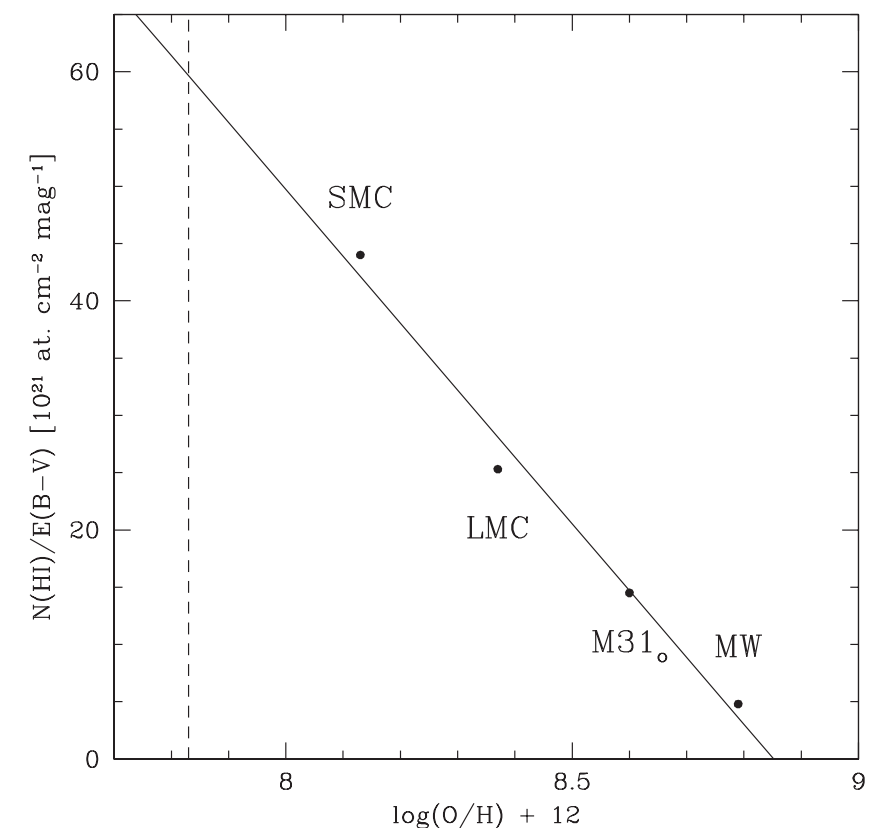

Fig. 8. Gas-to-dust ratio versus $\log (\mathrm{O} / \mathrm{H})+12$ relation. Data points are labeled (see Sect. 6.1 for details). The solid line is the linear leastsquares fit, and the vertical dashed line indicates the WLM abundance from Lee et al. (2005).

We obtained a $\mathrm{C} / \mathrm{M}$ ratio of $\sim 0.6 \pm 0.1$ and a peak of the carbon stars luminosity function of $\left\langle M_{K_{\mathrm{S}}}\right\rangle=-7.51 \mathrm{mag}$ for the WLM.

To measure the intrinsic reddening in WLM we used the H I map of WLM (Jackson et al. 2004), and we adopted a linear dependence of the gas-to-dust ratio on the metallicity: $N(\mathrm{H} \mathrm{I}) / E(B-V)=-(58 \pm 5) \times[\log (\mathrm{O} / \mathrm{H})+12]+(517 \pm 44)$. The gas-to-dust ratio for the WLM is $60 \pm 10\left[10^{21}\right.$ at. $\left.\mathrm{cm}^{-2} \mathrm{mag}^{-1}\right]$. This allows us to measure the extinction along the line of sight towards individual Cepheids and by the period-luminosity relation for the Cepheids (Gieren 1998), in the IR we determined the distance to the WLM $-(m-M)_{0}=24.84 \pm 0.14$ mag, similar to the previous estimates. To probe the significance of the used PL relation, we re-determined the distance modulus using the PL relation calibrated by Testa et al. (2006) for the Cepheids in two LMC young clusters. This results in a distance modulus between 24.76 and 25.00, depending on the assumed pulsation models. Finally, we fitted the surface brightness distribution along the main axis of WLM with an exponential law yielding

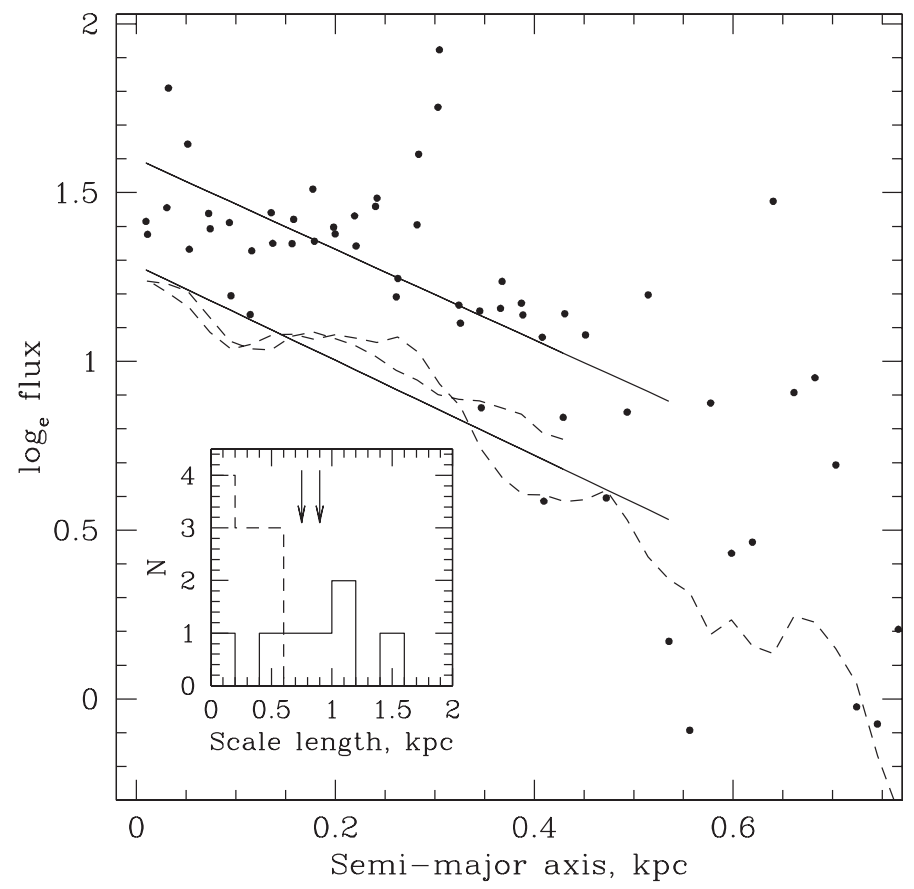

Fig. 9. Scale length of the WLM disk in $J$-band. The dots indicate the average values along the semi-major axis, the dashed line is the same for the median-averaged image. The solid lines are the fits. The inset shows the distribution of the scale lengths for the dwarf irregulars (solid line) and dwarf spheroidal galaxies (dashed lines) in the Local Group. The left arrow indicates our estimate and the right arrow shows the estimate of Fisher \& Tully (1975).

a scale length of $0.75 \pm 0.14 \mathrm{kpc}$, a typical value for the dwarf irregular galaxies.

Acknowledgements. This work obtained limited support by the grants F-1302/03 and VU-F-201/06 of the Bulgarian Scientific Foundation. Also, this publication makes use of data products from the Two Micron All Sky Survey, which is a joint project of the University of Massachusetts and the IR Processing and Analysis Center/California Institute of Technology, funded by the National Aeronautics and Space Administration and the National Science Foundation. This research has made use of the SIMBAD database, operated at CDS, Strasbourg, France. The authors thank Dr. M.-R. Cioni for the useful comments and the anonymous referee for the help in improving the article.

\section{References}

Ables, H. D., \& Ables, P. G. 1977, ApJS, 34, 245

Albert, L., Demers, S., \& Kunkel, W. E. 2000, ApJ, 119, 2780 
Battinelli, P., \& Demers, S. 2004, A\&A, 416, 111

Battinelli, P., \& Demers, S. 2005, A\&A, 434, 657

Bertelli, G., Bressan, A., Chiosi, C., Fagotto, F., \& Nasi, E. 1994, A\&AS, 106, 275

Bohlin, R., Savage, B.,\& Drake, J. 1978, ApJ, 224, 132

Cioni, M.-R. L., \& Habing, H. J. 2003, A\&A, 402, 133

Cioni, M.-R. L., \& Habing, H. J. 2005, A\&A, 429, 837

Cook, K. H., Aaronson, M., \& Norris, J. 1986, ApJ, 305, 634

Cuillandre, J., Lequeux, J., Allen, R. J., Mellier, Y., Bertin, E. 2001, ApJ, 554, 190

Dolphin, A. E. 2000, ApJ, 531, 804

Esteban, C., García-Rojas, J., Peimbert, M., et al. 2005, ApJS, 618, 95

Ferraro, F. R., Fusi Pecci, F., Tosi, M., \& Buonanno, R. 1989, MNRAS, 241, 433

Fisher, J. R., \& Tully, R. B. 1975, A\&A, 44, 151

Gieren, W. P., Fouque, P., \& Gomez, M. 1998, ApJ, 496, 17

Groenewegen, M. A. T. 2006, pnbm.conf., 108

Hodge, P. W., Dolphin, A. E., Smith, R. T., \& Mateo, M. 1999, ApJ, 521, 577

Iben, I. \& Renzini, A. 1983, ARA\&A, 21, 271

Ivanov, V. D., \& Borissova, J. 2002, 390, 937

Jackson, D. C., Skillman, E. D., Cannon, J. M., \& Côté, S. 2004, AJ, 128, 1219

Jakobsson, P., Hjorth, J., Fynbo, J. P. U., et al. 2004, A\&A, 427, 785
Junkkarinen, V., Cohen, R., Ross, D., et al. 2004, ApJ, 614, 658

Lee, H., Skillman, E. D., \& Venn, K. A. 2005, ApJ, 620, 223

Kang, A., Sohn, Y.-J., Rhee, J., et al. 2005, A\&A, 437, 61

Kang, A., Sohn, Y.-J., Kim, H.-I., et al. 2006, A\&A, 454, 727

Mateo, M. 1998, ARA\&A, 36, 435

Melotte, P. J. 1926, MNRAS, 86, 636

Minniti, D., \& Zijlstra, A. A. 1997, AJ, 114, 147

Mora, M., Minniti, D., Catelan, M., Zijlstra, A., \& Rejkuba, M. 2007, A\&A, in preparation

Rejkuba, M., Minniti, D., Gregg, M. D., et al. 2000 ApJ, 120, 801

Rieke, G. M., \& Lebofsky, M. J. 1985, ApJ, 288, 618

Russell, S. C., \& Dopita, M. A. 1990, ApJS, 74, 93

Sandage, A., \& Carlson, G. 1985, AJ, 90, 1464

Scalo. J. M., \& Miller, G. E. 1981, ApJ, 248, L65

Schlegel, D. J., Frankbeiner, D. P., \& Davis, M. 1998, ApJ, 500, 525

Smartt, S., Crowther, P., Dufton, P., et al. 2001, MNRAS, 325, 257

Sohn, Y.-J., Kang, A., Rhee, J., et al. 2006, ApJ, 445, 69

Testa, V., Ferraro, F. R., Ripepi, V., et al. 2006, MmSAI, 77, 263

Venn, K. A., Tolstoy, E., Kaufer, A., et al. 2003, AJ, 126, 1326

Wolf, M. 1909, Astron. Nachr., 183, 187 
A. T. Valcheva et al.: Near-IR view of WLM: carbon stars and C/M ratio, Online Material $p 1$

\section{Online Material}


A. T. Valcheva et al.: Near-IR view of WLM: carbon stars and C/M ratio, Online Material p 2

Table 1. Photometry of $555 \mathrm{WLM}$ stars with $J$ - and $K_{\mathrm{S}}$-band photometry (see Sect. 2 for details).

\begin{tabular}{|c|c|c|c|c|c|}
\hline$\overline{\mathrm{No}}$ & $\overline{\mathrm{RA}}$ & $\overline{\overline{\text { Dec }}}$ & 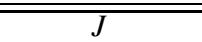 & $\overline{\bar{K}}$ & $\overline{\text { Note }}$ \\
\hline 1 & $00: 01: 45.62$ & $-15: 30: 06.6$ & $19.713(0.135)$ & $18.268(0.131)$ & M05 LC 120 \\
\hline 2 & $00: 01: 46.86$ & $-15: 29: 44.0$ & $18.150(0.047)$ & $16.737(0.058)$ & $\mathrm{C}$ \\
\hline 3 & $00: 01: 47.40$ & $-15: 29: 49.1$ & $19.762(0.119)$ & $18.376(0.155)$ & $\mathrm{C}$ \\
\hline 4 & $00: 01: 47.50$ & $-15: 27: 54.8$ & $19.222(0.073)$ & $18.402(0.140)$ & \\
\hline 5 & 00:01:47.99 & $-15: 27: 47.5$ & $19.408(0.092)$ & $17.487(0.065)$ & BD04 \\
\hline 6 & $00: 01: 48.20$ & $-15: 26: 49.0$ & $19.327(0.088)$ & $17.855(0.097)$ & $\mathrm{C}$ \\
\hline 7 & $00: 01: 48.34$ & $-15: 28: 08.5$ & $19.402(0.107)$ & $17.462(0.096)$ & $\mathrm{C}$ \\
\hline 8 & $00: 01: 48.42$ & $-15: 27: 08.6$ & $19.078(0.069)$ & $18.273(0.118)$ & \\
\hline 9 & 00:01:48.79 & $-15: 27: 35.2$ & $19.800(0.130)$ & $18.849(0.232)$ & \\
\hline 10 & 00:01:48.80 & $-15: 27: 16.8$ & $19.132(0.068)$ & $18.037(0.108)$ & BD04 \\
\hline 11 & 00:01:49.09 & $-15: 27: 06.3$ & $19.712(0.109)$ & $18.985(0.255)$ & \\
\hline 12 & 00:01:49.11 & $-15: 29: 03.3$ & $19.708(0.114)$ & $19.501(0.418)$ & \\
\hline 13 & $00: 01: 49.26$ & $-15: 26: 53.4$ & $13.177(0.029)$ & $12.683(0.004)$ & \\
\hline 14 & $00: 01: 49.33$ & $-15: 28: 24.1$ & $19.495(0.092)$ & $18.920(0.253)$ & \\
\hline 15 & $00: 01: 49.50$ & $-15: 26: 06.4$ & $18.821(0.056)$ & $18.021(0.103)$ & \\
\hline 16 & $00: 01: 49.56$ & $-15: 27: 31.0$ & $15.615(0.222)$ & $14.631(0.042)$ & G \\
\hline 17 & $00: 01: 49.57$ & $-15: 28: 00.6$ & $18.921(0.075)$ & $17.781(0.090)$ & \\
\hline 18 & 00:01:49.79 & $-15: 29: 42.1$ & $19.211(0.076)$ & $18.764(0.217)$ & \\
\hline 19 & 00:01:49.88 & $-15: 26: 32.3$ & $18.833(0.091)$ & $17.404(0.090)$ & $\mathrm{C}$ \\
\hline 20 & 00:01:49.94 & $-15: 26: 20.7$ & $19.196(0.076)$ & $18.640(0.192)$ & \\
\hline 21 & $00: 01: 50.10$ & $-15: 26: 25.5$ & $18.590(0.053)$ & $18.563(0.172)$ & \\
\hline 22 & $00: 01: 50.31$ & $-15: 26: 34.8$ & $19.815(0.116)$ & $18.808(0.223)$ & \\
\hline 23 & $00: 01: 50.56$ & $-15: 28: 30.7$ & $18.327(0.045)$ & $16.757(0.034)$ & BD04 \\
\hline 24 & 00:01:50.67 & $-15: 27: 31.9$ & $19.120(0.082)$ & $17.820(0.084)$ & BD04 \\
\hline 25 & $00: 01: 50.68$ & $-15: 28: 16.7$ & $18.818(0.078)$ & $17.285(0.075)$ & $\mathrm{C}$ \\
\hline 26 & $00: 01: 50.83$ & $-15: 25: 33.6$ & $19.773(0.126)$ & $18.734(0.240)$ & \\
\hline 27 & $00: 01: 50.84$ & $-15: 29: 47.0$ & $18.855(0.049)$ & $17.596(0.074)$ & $\mathrm{C}$ \\
\hline 28 & $00: 01: 50.85$ & $-15: 26: 51.4$ & $18.992(0.062)$ & $17.934(0.092)$ & BD04 \\
\hline 29 & 00:01:51.01 & $-15: 26: 14.4$ & $18.235(0.034)$ & $17.572(0.071)$ & \\
\hline 30 & 00:01:51.02 & $-15: 27: 00.0$ & $19.603(0.098)$ & $18.085(0.112)$ & $\mathrm{C}$ \\
\hline 31 & $00: 01: 51.13$ & $-15: 26: 08.1$ & $19.519(0.108)$ & $18.930(0.262)$ & \\
\hline 32 & $00: 01: 51.14$ & $-15: 30: 08.3$ & $19.340(0.118)$ & $17.619(0.096)$ & $\mathrm{C}$ \\
\hline 33 & $00: 01: 51.15$ & $-15: 26: 33.8$ & $19.423(0.110)$ & $17.864(0.099)$ & $\mathrm{C}$ \\
\hline 34 & $00: 01: 51.17$ & $-15: 25: 57.7$ & $19.778(0.132)$ & $18.743(0.201)$ & \\
\hline 35 & 00:01:51.17 & $-15: 27: 13.1$ & $18.922(0.068)$ & $17.671(0.073)$ & $\mathrm{C}$ \\
\hline 36 & $00: 01: 51.33$ & $-15: 29: 13.2$ & $20.066(0.145)$ & $19.286(0.337)$ & \\
\hline 37 & 00:01:51.38 & $-15: 27: 37.5$ & $19.906(0.145)$ & $19.070(0.270)$ & \\
\hline 38 & $00: 01: 51.38$ & $-15: 29: 06.6$ & $16.753(0.033)$ & $16.035(0.020)$ & \\
\hline 39 & $00: 01: 51.40$ & $-15: 28: 55.2$ & $18.536(0.045)$ & $17.075(0.056)$ & $\mathrm{C}$ \\
\hline 40 & $00: 01: 51.48$ & $-15: 28: 37.5$ & $19.776(0.130)$ & $19.001(0.263)$ & \\
\hline 41 & $00: 01: 51.53$ & $-15: 28: 50.4$ & $19.572(0.098)$ & $18.812(0.208)$ & \\
\hline 42 & $00: 01: 51.56$ & $-15: 28: 10.2$ & $18.777(0.062)$ & $18.002(0.106)$ & \\
\hline 43 & $00: 01: 51.67$ & $-15: 25: 36.3$ & $19.515(0.093)$ & $17.528(0.065)$ & BD04 \\
\hline 44 & $00: 01: 51.75$ & $-15: 27: 40.3$ & $19.117(0.080)$ & $18.256(0.124)$ & \\
\hline 45 & $00: 01: 52.02$ & $-15: 28: 56.5$ & $19.692(0.110)$ & $18.824(0.231)$ & \\
\hline 46 & $00: 01: 52.11$ & $-15: 26: 29.7$ & $18.951(0.062)$ & $18.000(0.105)$ & \\
\hline 47 & $00: 01: 52.20$ & $-15: 29: 50.8$ & $19.494(0.104)$ & $17.803(0.090)$ & $\mathrm{C}$ \\
\hline 48 & $00: 01: 52.23$ & $-15: 25: 19.0$ & $19.065(0.078)$ & $17.865(0.113)$ & \\
\hline 49 & $00: 01: 52.23$ & $-15: 28: 17.2$ & $19.973(0.145)$ & $17.966(0.104)$ & $\mathrm{C}$ \\
\hline 50 & $00: 01: 52.33$ & $-15: 26: 07.5$ & $19.867(0.128)$ & $19.471(0.393)$ & \\
\hline 51 & $00: 01: 52.33$ & $-15: 27: 59.7$ & $19.239(0.066)$ & $18.510(0.163)$ & \\
\hline 52 & $00: 01: 52.38$ & $-15: 29: 50.5$ & $18.643(0.050)$ & $16.911(0.040)$ & BD04 \\
\hline 53 & $00: 01: 52.42$ & $-15: 25: 55.2$ & $18.831(0.061)$ & $17.118(0.046)$ & BD04 \\
\hline 54 & $00: 01: 52.47$ & $-15: 25: 39.2$ & $18.152(0.176)$ & $16.300(0.058)$ & $\mathrm{C}$ \\
\hline 55 & $00: 01: 52.53$ & $-15: 28: 47.7$ & $19.924(0.139)$ & $19.274(0.365)$ & \\
\hline 56 & $00: 01: 52.57$ & $-15: 26: 20.8$ & $18.428(0.052)$ & $16.908(0.039)$ & BD04 \\
\hline 57 & $00: 01: 52.57$ & $-15: 27: 02.8$ & $19.822(0.134)$ & $18.755(0.209)$ & \\
\hline 58 & $00: 01: 52.73$ & $-15: 26: 38.8$ & $19.123(0.092)$ & $17.962(0.123)$ & \\
\hline 59 & $00: 01: 52.78$ & $-15: 28: 07.8$ & $19.986(0.146)$ & $18.899(0.255)$ & \\
\hline 60 & $00: 01: 52.80$ & $-15: 26: 26.0$ & $19.585(0.100)$ & $18.682(0.199)$ & \\
\hline 61 & $00: 01: 52.85$ & $-15: 27: 28.3$ & $18.994(0.076)$ & $18.039(0.103)$ & \\
\hline 62 & $00: 01: 52.94$ & $-15: 26: 46.8$ & $19.235(0.085)$ & $18.573(0.165)$ & \\
\hline 63 & $00: 01: 52.94$ & $-15: 27: 49.4$ & $19.454(0.107)$ & $18.523(0.167)$ & \\
\hline 64 & 00:01:52.98 & $-15: 28: 56.8$ & $19.457(0.084)$ & $18.990(0.275)$ & \\
\hline 65 & 00:01:53.12 & $-15: 29: 22.1$ & $19.355(0.082)$ & $18.674(0.187)$ & \\
\hline
\end{tabular}


Table 1. continued.

\begin{tabular}{|c|c|c|c|c|c|}
\hline No & $\overline{\overline{\mathrm{RA}}}$ & $\overline{\overline{\text { Dec }}}$ & $\overline{\bar{J}}$ & $\overline{K_{\mathrm{S}}}$ & Note \\
\hline 66 & $00: 01: 53.17$ & $-15: 28: 14.1$ & $16.650(0.026)$ & $15.862(0.018)$ & \\
\hline 67 & 00:01:53.22 & $-15: 28: 40.3$ & $17.830(0.036)$ & $17.816(0.091)$ & \\
\hline 68 & 00:01:53.27 & $-15: 29: 25.6$ & $17.155(0.026)$ & $16.403(0.026)$ & \\
\hline 69 & 00:01:53.28 & $-15: 26: 33.7$ & $19.033(0.068)$ & $18.155(0.118)$ & \\
\hline 70 & 00:01:53.32 & $-15: 26: 43.8$ & $18.895(0.063)$ & $17.895(0.086)$ & \\
\hline 71 & 00:01:53.39 & $-15: 27: 07.4$ & $18.923(0.060)$ & $17.807(0.085)$ & \\
\hline 72 & 00:01:53.45 & $-15: 28: 33.4$ & $19.831(0.134)$ & $18.538(0.165)$ & $\mathrm{C}$ \\
\hline 73 & 00:01:53.54 & $-15: 28: 13.7$ & $17.765(0.053)$ & $16.228(0.066)$ & $\mathrm{C}$ \\
\hline 74 & 00:01:53.54 & $-15: 29: 16.7$ & $19.434(0.088)$ & $18.808(0.230)$ & \\
\hline 75 & 00:01:53.55 & $-15: 27: 16.5$ & $18.765(0.044)$ & $17.870(0.093)$ & \\
\hline 76 & 00:01:53.55 & $-15: 30: 04.9$ & $19.158(0.069)$ & $17.996(0.111)$ & \\
\hline 77 & 00:01:53.57 & $-15: 27: 55.2$ & $18.905(0.057)$ & $18.136(0.114)$ & \\
\hline 78 & 00:01:53.62 & $-15: 28: 23.0$ & $17.891(0.042)$ & $17.281(0.055)$ & \\
\hline 79 & 00:01:53.66 & $-15: 28: 19.4$ & $20.518(0.222)$ & $19.506(0.382)$ & \\
\hline 80 & 00:01:53.67 & $-15: 28: 21.0$ & $20.209(0.202)$ & $18.949(0.244)$ & \\
\hline 81 & 00:01:53.70 & $-15: 28: 37.2$ & $18.120(0.039)$ & $17.440(0.063)$ & \\
\hline 82 & 00:01:53.71 & $-15: 28: 15.3$ & $19.235(0.081)$ & $18.390(0.148)$ & \\
\hline 83 & 00:01:53.77 & $-15: 28: 50.1$ & $15.441(0.028)$ & $14.667(0.010)$ & \\
\hline 84 & 00:01:53.83 & $-15: 26: 39.9$ & $16.655(0.028)$ & $15.984(0.020)$ & \\
\hline 85 & 00:01:53.83 & $-15: 29: 39.7$ & $19.684(0.121)$ & $18.822(0.221)$ & \\
\hline 86 & 00:01:53.87 & $-15: 27: 03.5$ & $19.664(0.119)$ & $19.219(0.341)$ & \\
\hline 87 & 00:01:53.90 & $-15: 28: 18.5$ & $19.249(0.064)$ & $17.810(0.085)$ & $\mathrm{C}$ \\
\hline 88 & 00:01:53.94 & $-15: 27: 01.7$ & $19.380(0.086)$ & $18.516(0.167)$ & \\
\hline 89 & 00:01:53.96 & $-15: 26: 34.8$ & $18.964(0.067)$ & $17.849(0.101)$ & BD04 \\
\hline 90 & 00:01:54.02 & $-15: 29: 12.1$ & $19.092(0.071)$ & $16.905(0.042)$ & BD04 \\
\hline 91 & 00:01:54.03 & $-15: 28: 39.2$ & $19.411(0.102)$ & $19.220(0.326)$ & \\
\hline 92 & 00:01:54.04 & $-15: 29: 07.6$ & $18.171(0.037)$ & $16.749(0.050)$ & $\mathrm{C}$ \\
\hline 93 & 00:01:54.06 & $-15: 25: 31.1$ & $19.299(0.076)$ & $17.658(0.094)$ & $\mathrm{C}$ \\
\hline 94 & 00:01:54.10 & $-15: 27: 25.3$ & $19.785(0.141)$ & $18.052(0.105)$ & $\mathrm{C}$ \\
\hline 95 & $00: 01: 54.13$ & $-15: 25: 55.8$ & $19.876(0.112)$ & $18.436(0.142)$ & $\mathrm{C}$ \\
\hline 96 & 00:01:54.15 & $-15: 25: 37.9$ & $20.154(0.161)$ & $18.609(0.185)$ & $\mathrm{C}$ \\
\hline 97 & 00:01:54.16 & $-15: 28: 01.5$ & $19.772(0.132)$ & $18.847(0.218)$ & \\
\hline 98 & 00:01:54.19 & $-15: 28: 15.6$ & $19.236(0.080)$ & $18.434(0.158)$ & \\
\hline 99 & 00:01:54.25 & $-15: 28: 32.3$ & $15.476(0.030)$ & $14.817(0.009)$ & \\
\hline 100 & 00:01:54.27 & $-15: 29: 13.8$ & $18.221(0.050)$ & $16.639(0.051)$ & $\mathrm{C}$ \\
\hline 101 & 00:01:54.28 & $-15: 28: 22.2$ & $19.198(0.096)$ & $18.165(0.115)$ & \\
\hline 102 & 00:01:54.31 & $-15: 27: 53.1$ & $18.761(0.058)$ & $17.779(0.084)$ & \\
\hline 103 & 00:01:54.41 & $-15: 26: 30.3$ & $18.766(0.056)$ & $18.079(0.106)$ & \\
\hline 104 & 00:01:54.42 & $-15: 27: 18.0$ & $18.325(0.053)$ & $16.613(0.032)$ & BD04 \\
\hline 105 & 00:01:54.48 & $-15: 27: 46.5$ & $19.059(0.080)$ & $18.204(0.126)$ & \\
\hline 106 & 00:01:54.53 & $-15: 26: 21.8$ & $19.923(0.141)$ & $18.839(0.216)$ & \\
\hline 107 & 00:01:54.53 & $-15: 28: 44.0$ & $18.830(0.067)$ & $18.167(0.133)$ & \\
\hline 108 & 00:01:54.57 & $-15: 27: 54.8$ & $18.867(0.067)$ & $17.677(0.079)$ & BD04 \\
\hline 109 & 00:01:54.65 & $-15: 27: 47.5$ & $20.079(0.157)$ & $19.499(0.418)$ & \\
\hline 110 & 00:01:54.66 & $-15: 26: 04.2$ & $18.620(0.055)$ & $17.282(0.057)$ & BD04 \\
\hline 111 & 00:01:54.71 & $-15: 26: 07.4$ & $18.652(0.050)$ & $17.673(0.080)$ & \\
\hline 112 & 00:01:54.71 & $-15: 26: 23.5$ & $19.668(0.114)$ & $18.301(0.133)$ & $\mathrm{C}$ \\
\hline 113 & 00:01:54.71 & $-15: 26: 26.6$ & $18.857(0.072)$ & $17.438(0.065)$ & $\mathrm{C}$ \\
\hline 114 & 00:01:54.76 & $-15: 26: 53.9$ & $19.344(0.095)$ & $18.294(0.136)$ & \\
\hline 115 & 00:01:54.80 & $-15: 28: 37.0$ & $18.841(0.058)$ & $17.945(0.097)$ & \\
\hline 116 & 00:01:54.81 & $-15: 28: 17.5$ & $18.759(0.055)$ & $17.443(0.064)$ & BD04 \\
\hline 117 & 00:01:54.87 & $-15: 28: 46.2$ & $18.805(0.057)$ & $17.073(0.059)$ & $\mathrm{C}$ \\
\hline 118 & 00:01:54.89 & $-15: 27: 52.4$ & $19.896(0.132)$ & $18.985(0.262)$ & \\
\hline 119 & 00:01:54.89 & $-15: 28: 55.4$ & $19.610(0.106)$ & $18.841(0.215)$ & \\
\hline 120 & 00:01:54.98 & $-15: 29: 32.5$ & $17.715(0.077)$ & $15.943(0.092)$ & $\mathrm{C}$ \\
\hline 121 & 00:01:55.04 & $-15: 26: 52.1$ & $20.067(0.157)$ & $19.437(0.409)$ & \\
\hline 122 & 00:01:55.04 & $-15: 28: 18.7$ & $18.573(0.051)$ & $17.594(0.069)$ & \\
\hline 123 & 00:01:55.05 & $-15: 26: 04.7$ & $19.684(0.139)$ & $18.836(0.256)$ & \\
\hline 124 & 00:01:55.05 & $-15: 27: 20.5$ & $18.692(0.060)$ & $17.914(0.097)$ & \\
\hline 125 & 00:01:55.08 & $-15: 26: 49.1$ & $19.513(0.098)$ & $18.628(0.208)$ & \\
\hline 126 & 00:01:55.10 & $-15: 27: 01.1$ & $19.835(0.131)$ & $16.666(0.030)$ & $\mathrm{C}$ \\
\hline 127 & 00:01:55.11 & $-15: 25: 54.5$ & $20.179(0.150)$ & $19.348(0.376)$ & SC85 V15 \\
\hline 128 & 00:01:55.16 & $-15: 29: 49.2$ & $19.436(0.091)$ & $18.053(0.120)$ & $\mathrm{C}$ \\
\hline
\end{tabular}


Table 1. continued.

\begin{tabular}{|c|c|c|c|c|c|}
\hline$\overline{\mathrm{No}}$ & $\overline{\mathrm{RA}}$ & $\overline{\mathrm{Dec}}$ & $\bar{J}$ & $\overline{\overline{K_{\mathrm{S}}}}$ & Note \\
\hline 129 & $00: 01: 55.20$ & $-15: 26: 22.0$ & $20.209(0.161)$ & $16.950(0.041)$ & $\mathrm{C}$ \\
\hline 130 & 00:01:55.26 & $-15: 26: 06.2$ & $18.879(0.056)$ & $17.794(0.084)$ & \\
\hline 131 & 00:01:55.30 & $-15: 25: 51.8$ & $19.738(0.099)$ & $19.023(0.266)$ & \\
\hline 132 & 00:01:55.37 & $-15: 27: 10.6$ & $17.370(0.029)$ & $16.600(0.029)$ & \\
\hline 133 & 00:01:55.38 & $-15: 27: 31.8$ & $19.012(0.077)$ & $18.426(0.170)$ & \\
\hline 134 & 00:01:55.39 & $-15: 28: 06.7$ & $19.047(0.074)$ & $18.129(0.113)$ & \\
\hline 135 & 00:01:55.39 & $-15: 28: 47.3$ & $19.381(0.095)$ & $18.068(0.116)$ & $\mathrm{C}$ \\
\hline 136 & 00:01:55.42 & $-15: 29: 29.4$ & $18.456(0.045)$ & $17.948(0.109)$ & \\
\hline 137 & 00:01:55.44 & $-15: 26: 54.6$ & $19.105(0.069)$ & $18.134(0.107)$ & \\
\hline 138 & 00:01:55.47 & $-15: 25: 52.1$ & $17.644(0.034)$ & $16.714(0.033)$ & \\
\hline 139 & 00:01:55.50 & $-15: 27: 15.6$ & $17.535(0.038)$ & $16.775(0.037)$ & \\
\hline 140 & 00:01:55.50 & $-15: 27: 25.4$ & $19.421(0.095)$ & $18.539(0.179)$ & \\
\hline 141 & 00:01:55.53 & $-15: 26: 17.1$ & $18.501(0.046)$ & $17.083(0.044)$ & BD04 \\
\hline 142 & 00:01:55.53 & $-15: 26: 48.3$ & $18.609(0.059)$ & $17.433(0.059)$ & BD04 \\
\hline 143 & 00:01:55.53 & $-15: 28: 53.4$ & $18.075(0.043)$ & $17.186(0.048)$ & \\
\hline 144 & 00:01:55.56 & $-15: 27: 37.1$ & $19.123(0.068)$ & $18.140(0.116)$ & \\
\hline 145 & 00:01:55.56 & $-15: 28: 54.7$ & $19.553(0.103)$ & $18.591(0.181)$ & \\
\hline 146 & 00:01:55.58 & $-15: 28: 37.7$ & $18.207(0.042)$ & $17.390(0.062)$ & \\
\hline 147 & 00:01:55.60 & $-15: 27: 47.1$ & $19.242(0.073)$ & $18.640(0.191)$ & \\
\hline 148 & 00:01:55.60 & $-15: 27: 53.4$ & $19.675(0.134)$ & $19.279(0.355)$ & \\
\hline 149 & 00:01:55.62 & $-15: 25: 27.7$ & $19.653(0.137)$ & $18.864(0.270)$ & \\
\hline 150 & 00:01:55.62 & $-15: 30: 01.5$ & $19.821(0.142)$ & $19.292(0.361)$ & \\
\hline 151 & 00:01:55.69 & $-15: 27: 02.3$ & $18.736(0.056)$ & $17.574(0.068)$ & BD04 \\
\hline 152 & 00:01:55.71 & $-15: 26: 24.6$ & $19.022(0.057)$ & $17.521(0.063)$ & BD04 \\
\hline 153 & 00:01:55.77 & $-15: 28: 39.4$ & $16.783(0.029)$ & $15.995(0.017)$ & \\
\hline 154 & 00:01:55.79 & $-15: 29: 22.6$ & $20.316(0.241)$ & $18.803(0.228)$ & \\
\hline 155 & 00:01:55.81 & $-15: 25: 56.4$ & $18.728(0.052)$ & $17.498(0.070)$ & BD04 \\
\hline 156 & 00:01:55.83 & $-15: 28: 12.7$ & $19.593(0.116)$ & $18.599(0.177)$ & \\
\hline 157 & 00:01:55.86 & $-15: 27: 30.2$ & $19.089(0.067)$ & $18.477(0.144)$ & \\
\hline 158 & 00:01:55.87 & $-15: 25: 31.4$ & $19.538(0.136)$ & $18.837(0.225)$ & \\
\hline 159 & 00:01:55.89 & $-15: 29: 04.7$ & $18.291(0.042)$ & $17.631(0.081)$ & \\
\hline 160 & 00:01:55.93 & $-15: 29: 33.0$ & $19.060(0.074)$ & $18.211(0.143)$ & \\
\hline 161 & 00:01:55.94 & $-15: 26: 44.5$ & $18.468(0.055)$ & $17.032(0.045)$ & BD04 \\
\hline 162 & 00:01:56.00 & $-15: 27: 03.5$ & $19.050(0.070)$ & $17.979(0.114)$ & \\
\hline 163 & 00:01:56.00 & $-15: 28: 44.6$ & $19.199(0.080)$ & $18.585(0.169)$ & \\
\hline 164 & 00:01:56.01 & $-15: 28: 04.3$ & $19.887(0.253)$ & $18.667(0.205)$ & $\mathrm{C}$ \\
\hline 165 & 00:01:56.02 & $-15: 25: 57.0$ & $19.651(0.105)$ & $18.810(0.210)$ & \\
\hline 166 & 00:01:56.10 & $-15: 26: 42.6$ & $19.850(0.151)$ & $18.114(0.125)$ & $\mathrm{C}$ \\
\hline 167 & 00:01:56.10 & $-15: 29: 06.2$ & $18.708(0.061)$ & $17.447(0.063)$ & BD04 \\
\hline 168 & 00:01:56.12 & $-15: 28: 05.8$ & $19.055(0.072)$ & $17.789(0.097)$ & BD04 \\
\hline 169 & 00:01:56.16 & $-15: 27: 10.3$ & $19.148(0.072)$ & $18.636(0.186)$ & \\
\hline 170 & 00:01:56.18 & $-15: 29: 21.2$ & $18.800(0.054)$ & $18.263(0.149)$ & \\
\hline 171 & 00:01:56.21 & $-15: 27: 35.8$ & $18.641(0.055)$ & $17.020(0.047)$ & BD04 \\
\hline 172 & 00:01:56.27 & $-15: 27: 51.5$ & $19.902(0.139)$ & $18.899(0.209)$ & \\
\hline 173 & 00:01:56.28 & $-15: 26: 35.7$ & $17.588(0.031)$ & $17.077(0.042)$ & \\
\hline 174 & 00:01:56.28 & $-15: 28: 14.8$ & $19.061(0.069)$ & $18.144(0.147)$ & \\
\hline 175 & 00:01:56.29 & $-15: 26: 45.6$ & $18.790(0.069)$ & $17.948(0.096)$ & \\
\hline 176 & 00:01:56.29 & $-15: 29: 17.8$ & $18.915(0.064)$ & $17.992(0.102)$ & \\
\hline 177 & 00:01:56.34 & $-15: 25: 26.5$ & $20.146(0.177)$ & $19.269(0.445)$ & \\
\hline 178 & 00:01:56.34 & $-15: 28: 14.8$ & $18.775(0.057)$ & $17.868(0.111)$ & \\
\hline 179 & 00:01:56.37 & $-15: 28: 49.9$ & $19.488(0.094)$ & $18.496(0.172)$ & \\
\hline 180 & 00:01:56.39 & $-15: 25: 50.1$ & $19.564(0.108)$ & $19.261(0.338)$ & \\
\hline 181 & 00:01:56.39 & $-15: 26: 49.7$ & $19.759(0.124)$ & $18.881(0.228)$ & \\
\hline 182 & 00:01:56.41 & $-15: 25: 52.5$ & $19.404(0.101)$ & $18.593(0.169)$ & \\
\hline 183 & 00:01:56.43 & $-15: 28: 52.3$ & $19.920(0.134)$ & $19.053(0.296)$ & \\
\hline 184 & 00:01:56.44 & $-15: 28: 47.2$ & $18.679(0.051)$ & $17.006(0.041)$ & $\mathrm{BD}$ \\
\hline 185 & 00:01:56.45 & $-15: 25: 22.8$ & $18.741(0.074)$ & $17.516(0.078)$ & BD04 \\
\hline 186 & 00:01:56.45 & $-15: 27: 03.7$ & $18.516(0.049)$ & $18.676(0.201)$ & \\
\hline 187 & 00:01:56.45 & $-15: 27: 08.3$ & $19.401(0.099)$ & $18.932(0.248)$ & \\
\hline 188 & 00:01:56.49 & $-15: 25: 45.9$ & $19.876(0.137)$ & $19.624(0.493)$ & \\
\hline 189 & 00:01:56.52 & $-15: 26: 02.8$ & $19.912(0.127)$ & $19.152(0.290)$ & \\
\hline 190 & 00:01:56.54 & $-15: 27: 39.9$ & $19.792(0.102)$ & $18.830(0.235)$ & \\
\hline 191 & 00:01:56.62 & $-15: 26: 42.3$ & $18.970(0.070)$ & $18.002(0.113)$ & \\
\hline 192 & 00:01:56.64 & $-15: 27: 48.1$ & $19.358(0.100)$ & $17.948(0.102)$ & BD04 \\
\hline 193 & 00:01:56.67 & $-15: 28: 31.8$ & $18.567(0.059)$ & $17.990(0.112)$ & \\
\hline 194 & 00:01:56.68 & $-15: 25: 47.0$ & $18.586(0.049)$ & $17.491(0.068)$ & \\
\hline
\end{tabular}


Table 1. continued.

\begin{tabular}{|c|c|c|c|c|c|}
\hline No & $\overline{\mathrm{RA}}$ & $\overline{\mathrm{Dec}}$ & $\bar{J}$ & $\overline{K_{\mathrm{S}}}$ & Note \\
\hline 195 & $00: 01: 56.68$ & $-15: 27: 41.9$ & $19.428(0.099)$ & $18.451(0.162)$ & \\
\hline 196 & 00:01:56.69 & $-15: 28: 03.9$ & $18.380(0.048)$ & $18.059(0.124)$ & \\
\hline 197 & 00:01:56.72 & $-15: 28: 17.5$ & $19.035(0.065)$ & $18.434(0.175)$ & \\
\hline 198 & 00:01:56.73 & $-15: 26: 03.7$ & $18.810(0.065)$ & $18.062(0.111)$ & \\
\hline 199 & 00:01:56.73 & $-15: 28: 40.5$ & $14.625(0.024)$ & $13.656(0.008)$ & SC85 28 LC \\
\hline 200 & 00:01:56.74 & $-15: 26: 58.6$ & $18.809(0.061)$ & $18.112(0.123)$ & \\
\hline 201 & 00:01:56.78 & $-15: 27: 09.2$ & $19.321(0.093)$ & $18.398(0.147)$ & \\
\hline 202 & 00:01:56.86 & $-15: 28: 38.0$ & $19.879(0.147)$ & $18.196(0.126)$ & $\mathrm{C}$ \\
\hline 203 & 00:01:56.87 & $-15: 27: 10.7$ & $18.575(0.056)$ & $17.892(0.101)$ & \\
\hline 204 & 00:01:56.87 & $-15: 28: 50.1$ & $17.500(0.037)$ & $16.510(0.030)$ & \\
\hline 205 & 00:01:56.89 & $-15: 25: 26.2$ & $18.370(0.045)$ & $17.228(0.067)$ & BD04 \\
\hline 206 & 00:01:56.90 & $-15: 27: 05.4$ & $19.360(0.080)$ & $19.178(0.286)$ & \\
\hline 207 & 00:01:56.90 & $-15: 28: 30.3$ & $19.135(0.072)$ & $18.365(0.129)$ & \\
\hline 208 & 00:01:56.92 & $-15: 26: 12.3$ & $18.693(0.057)$ & $17.921(0.095)$ & \\
\hline 209 & 00:01:56.94 & $-15: 30: 05.3$ & $17.581(0.050)$ & $15.649(0.059)$ & $\mathrm{C}$ \\
\hline 210 & 00:01:56.99 & $-15: 29: 55.4$ & $16.131(0.023)$ & $17.407(0.149)$ & SC85 11 LC \\
\hline 211 & 00:01:57.03 & $-15: 28: 22.6$ & $18.801(0.053)$ & $18.570(0.168)$ & \\
\hline 212 & 00:01:57.05 & $-15: 26: 38.0$ & $18.699(0.053)$ & $17.908(0.104)$ & \\
\hline 213 & 00:01:57.05 & $-15: 27: 50.2$ & $19.361(0.089)$ & $19.268(0.340)$ & \\
\hline 214 & 00:01:57.08 & $-15: 29: 07.9$ & $19.609(0.127)$ & $18.528(0.181)$ & \\
\hline 215 & 00:01:57.10 & $-15: 26: 17.6$ & $17.329(0.031)$ & $16.362(0.025)$ & \\
\hline 216 & 00:01:57.12 & $-15: 27: 17.3$ & $19.847(0.131)$ & $18.848(0.257)$ & \\
\hline 217 & 00:01:57.19 & $-15: 28: 35.5$ & $19.349(0.088)$ & $18.643(0.180)$ & \\
\hline 218 & 00:01:57.20 & $-15: 26: 43.9$ & $18.881(0.063)$ & $18.248(0.128)$ & \\
\hline 219 & 00:01:57.20 & $-15: 27: 10.5$ & $19.199(0.089)$ & $18.584(0.177)$ & \\
\hline 220 & 00:01:57.22 & $-15: 27: 22.8$ & $19.789(0.118)$ & $18.780(0.218)$ & \\
\hline 221 & 00:01:57.27 & $-15: 26: 08.2$ & $18.901(0.060)$ & $18.003(0.108)$ & \\
\hline 222 & 00:01:57.33 & $-15: 25: 52.5$ & $19.565(0.100)$ & $18.435(0.164)$ & \\
\hline 223 & 00:01:57.33 & $-15: 26: 20.8$ & $18.728(0.052)$ & $17.799(0.087)$ & \\
\hline 224 & 00:01:57.37 & $-15: 26: 32.9$ & $18.806(0.063)$ & $17.827(0.093)$ & \\
\hline 225 & 00:01:57.38 & $-15: 29: 22.4$ & $17.740(0.034)$ & $16.610(0.031)$ & \\
\hline 226 & 00:01:57.39 & $-15: 26: 26.4$ & $19.490(0.111)$ & $19.008(0.252)$ & \\
\hline 227 & 00:01:57.39 & $-15: 27: 50.3$ & $19.404(0.096)$ & $18.578(0.183)$ & \\
\hline 228 & 00:01:57.39 & $-15: 28: 27.5$ & $20.057(0.150)$ & $18.998(0.243)$ & \\
\hline 229 & 00:01:57.40 & $-15: 25: 59.6$ & $18.332(0.043)$ & $17.568(0.078)$ & \\
\hline 230 & 00:01:57.46 & $-15: 26: 42.8$ & $19.111(0.084)$ & $18.371(0.149)$ & \\
\hline 231 & 00:01:57.49 & $-15: 29: 06.8$ & $19.522(0.107)$ & $18.653(0.191)$ & \\
\hline 232 & 00:01:57.51 & $-15: 29: 14.4$ & $18.978(0.066)$ & $18.190(0.127)$ & \\
\hline 233 & 00:01:57.51 & $-15: 29: 17.0$ & $16.520(0.026)$ & $15.648(0.015)$ & SC85 16 LC \\
\hline 234 & 00:01:57.52 & $-15: 26: 12.9$ & $18.954(0.065)$ & $17.902(0.089)$ & \\
\hline 235 & 00:01:57.53 & $-15: 26: 11.4$ & $19.405(0.100)$ & $18.456(0.149)$ & \\
\hline 236 & 00:01:57.54 & $-15: 26: 03.1$ & $18.584(0.060)$ & $17.830(0.098)$ & \\
\hline 237 & 00:01:57.54 & $-15: 27: 57.5$ & $18.289(0.052)$ & $17.611(0.071)$ & \\
\hline 238 & 00:01:57.55 & $-15: 29: 02.5$ & $18.510(0.050)$ & $17.617(0.078)$ & \\
\hline 239 & 00:01:57.58 & $-15: 26: 55.1$ & $19.684(0.120)$ & $18.824(0.265)$ & \\
\hline 240 & 00:01:57.59 & $-15: 26: 38.0$ & $19.903(0.153)$ & $19.150(0.292)$ & \\
\hline 241 & 00:01:57.63 & $-15: 29: 11.7$ & $18.472(0.048)$ & $16.856(0.040)$ & BD04 \\
\hline 242 & 00:01:57.66 & $-15: 28: 50.1$ & $19.819(0.119)$ & $18.807(0.202)$ & \\
\hline 243 & 00:01:57.68 & $-15: 26: 37.2$ & $20.692(0.287)$ & $19.438(0.382)$ & \\
\hline 244 & 00:01:57.69 & $-15: 26: 42.0$ & $19.680(0.121)$ & $19.094(0.288)$ & \\
\hline 245 & 00:01:57.70 & $-15: 27: 04.9$ & $19.737(0.127)$ & $18.808(0.226)$ & \\
\hline 246 & 00:01:57.84 & $-15: 28: 45.4$ & $18.952(0.071)$ & $18.182(0.122)$ & \\
\hline 247 & 00:01:57.84 & $-15: 29: 02.9$ & $20.302(0.203)$ & $19.165(0.331)$ & \\
\hline 248 & 00:01:57.85 & $-15: 29: 25.0$ & $18.887(0.050)$ & $17.338(0.080)$ & $\mathrm{C}$ \\
\hline 249 & 00:01:57.86 & $-15: 28: 17.6$ & $19.876(0.137)$ & $19.308(0.406)$ & \\
\hline 250 & 00:01:57.86 & $-15: 29: 49.2$ & $18.965(0.057)$ & $18.305(0.154)$ & \\
\hline 251 & 00:01:57.87 & $-15: 25: 26.2$ & $20.016(0.151)$ & $18.844(0.257)$ & \\
\hline 252 & 00:01:57.87 & $-15: 26: 49.6$ & $19.086(0.074)$ & $18.436(0.148)$ & \\
\hline 253 & 00:01:57.88 & $-15: 27: 47.3$ & $18.389(0.051)$ & $17.764(0.088)$ & \\
\hline 254 & 00:01:57.89 & $-15: 28: 04.0$ & $17.234(0.029)$ & $16.392(0.025)$ & \\
\hline 255 & 00:01:57.95 & $-15: 28: 34.7$ & $19.169(0.083)$ & $18.271(0.147)$ & \\
\hline 256 & 00:01:58.00 & $-15: 27: 10.5$ & $19.053(0.060)$ & $18.265(0.136)$ & \\
\hline 257 & 00:01:58.02 & $-15: 28: 00.4$ & $18.703(0.048)$ & $16.922(0.046)$ & $\mathrm{C}$ \\
\hline 258 & 00:01:58.04 & $-15: 29: 00.6$ & $19.786(0.118)$ & $18.844(0.279)$ & \\
\hline 259 & 00:01:58.07 & $-15: 27: 50.7$ & $19.729(0.126)$ & $18.748(0.228)$ & \\
\hline 260 & 00:01:58.07 & $-15: 28: 04.7$ & $20.744(0.310)$ & $17.875(0.096)$ & $\mathrm{C}$ \\
\hline
\end{tabular}


Table 1. continued.

\begin{tabular}{|c|c|c|c|c|c|}
\hline No & RA & Dec & $J$ & $K_{\mathrm{S}}$ & Note \\
\hline 261 & 00:01:58.08 & $-15: 27: 40.0$ & $13.649(0.025)$ & $12.915(0.006)$ & \\
\hline 262 & 00:01:58.09 & $-15: 28: 02.0$ & $18.058(0.039)$ & $17.298(0.053)$ & \\
\hline 263 & 00:01:58.10 & $-15: 26: 42.2$ & $19.472(0.089)$ & $18.752(0.188)$ & \\
\hline 264 & 00:01:58.11 & $-15: 26: 15.1$ & $19.762(0.126)$ & $19.064(0.251)$ & \\
\hline 265 & 00:01:58.11 & $-15: 27: 03.3$ & $19.129(0.076)$ & $18.492(0.185)$ & \\
\hline 266 & 00:01:58.12 & $-15: 25: 54.5$ & $19.208(0.183)$ & $17.366(0.077)$ & $\mathrm{C}$ \\
\hline 267 & 00:01:58.14 & $-15: 25: 37.4$ & $18.594(0.055)$ & $16.984(0.046)$ & $\mathrm{C}$ \\
\hline 268 & 00:01:58.16 & $-15: 25: 27.0$ & $19.149(0.079)$ & $17.839(0.107)$ & $\mathrm{C}$ \\
\hline 269 & 00:01:58.16 & $-15: 25: 54.7$ & $19.629(0.256)$ & $17.366(0.077)$ & $\mathrm{C}$ \\
\hline 270 & 00:01:58.17 & $-15: 27: 47.9$ & $20.312(0.211)$ & $19.518(0.424)$ & \\
\hline 271 & 00:01:58.19 & $-15: 30: 00.2$ & $18.171(0.035)$ & $16.236(0.025)$ & BD04 \\
\hline 272 & 00:01:58.20 & $-15: 29: 10.0$ & $19.003(0.063)$ & $17.737(0.084)$ & $\mathrm{C}$ \\
\hline 273 & 00:01:58.24 & $-15: 25: 53.5$ & $19.463(0.122)$ & $18.405(0.149)$ & \\
\hline 274 & 00:01:58.25 & $-15: 25: 52.5$ & $18.708(0.058)$ & $17.740(0.075)$ & \\
\hline 275 & 00:01:58.29 & $-15: 25: 40.3$ & $19.201(0.074)$ & $18.357(0.157)$ & \\
\hline 276 & 00:01:58.29 & $-15: 27: 55.3$ & $18.482(0.049)$ & $17.330(0.053)$ & BD04 \\
\hline 277 & 00:01:58.29 & $-15: 28: 07.8$ & $19.734(0.124)$ & $18.420(0.177)$ & $\mathrm{C}$ \\
\hline 278 & 00:01:58.36 & $-15: 27: 53.9$ & $19.957(0.160)$ & $18.999(0.271)$ & BD04 \\
\hline 279 & 00:01 & $-15: 25$ & $20.156(0.148)$ & $18.954(0.306)$ & \\
\hline 280 & 00:01:58.40 & $-15: 28: 00.6$ & $18.762(0.056)$ & $17.422(0.061)$ & $\mathrm{BD}$ \\
\hline 281 & 00:01:58.41 & $-15: 26: 25.5$ & $19.152(0.0$ & 18.215 & \\
\hline 282 & 00:01:58.41 & $-15: 29: 04.0$ & $19.226(0.077)$ & 18.101 & \\
\hline 283 & 00:01:58.42 & $-15: 25: 36.2$ & $19.505(0.099)$ & 18.455 & \\
\hline 284 & 00:01:58.44 & $-15: 27: 55.1$ & $19.286(0.091)$ & $18.494(0.170)$ & $\mathrm{BD}$ \\
\hline 285 & 00:01:58.46 & $-15: 26: 07.5$ & $18.764(0.048)$ & $17.653(0.069)$ & \\
\hline 286 & 00:01:58.47 & $-15: 26: 09.0$ & $19.612(0.107)$ & $17.965(0.097)$ & $\mathrm{C}$ \\
\hline 287 & 00:01:58.47 & $-15: 26: 32.8$ & $19.180(0.074)$ & $18.041(0.117)$ & BD04 \\
\hline 288 & 00:01:58.49 & $-15: 27: 20.3$ & $20.011(0.157)$ & $19.313(0.364)$ & \\
\hline 289 & 00:01:58.50 & $-15: 28: 45.0$ & $19.611(0.099)$ & $18.961(0.258)$ & \\
\hline 290 & 00:01:58.50 & $-15: 29: 16.5$ & $19.303(0.086)$ & $18.236(0$ & \\
\hline 291 & 00:0 & $-15: 27: 29.3$ & $18.507(0.043)$ & 17.372 & \\
\hline 292 & 00:0 & $-15: 25$ & 18.8 & 17.797 & BD04 \\
\hline 293 & 00:0 & $-15: 28: 08.2$ & 18.7 & 17.748 & \\
\hline 294 & $00: 0$ & $-15: 28: 03.9$ & 18. & 17.435 & \\
\hline 295 & 00:01:58.65 & $-15: 28: 40.5$ & 18. & 17. & \\
\hline 296 & 00:01:58.66 & $-15: 26: 43.1$ & 20. & 19. & \\
\hline 297 & 00:01:58.69 & $-15: 26: 15.4$ & $19.303(0.0$ & 18. & \\
\hline 298 & 00:01:58.69 & $-15: 26: 17.7$ & $19.820(0.137)$ & $18.880(0.235)$ & \\
\hline 299 & 00:01:58.70 & $-15: 28: 54.1$ & $19.527(0.098)$ & $18.608(0.171)$ & \\
\hline 300 & 00:01:58.72 & $-15: 28: 02.2$ & $18.645(0.046)$ & $16.906(0.055)$ & $\mathrm{C}$ \\
\hline 301 & 00:01:58.75 & $-15: 26: 02.7$ & $18.967(0.059)$ & $18.022(0.114)$ & \\
\hline 302 & 00:01:58.78 & $-15: 25: 35.1$ & $18.873(0.063)$ & $17.715(0.090)$ & \\
\hline 303 & 00:01:58.83 & $-15: 28: 15.9$ & $19.723(0.111)$ & $16.533(0.032)$ & $\mathrm{C}$ \\
\hline 304 & 00:01:58.83 & $-15: 29: 32.7$ & $18.599(0.048)$ & $17.359(0.063)$ & $\mathrm{D}$ \\
\hline 305 & 00:01:5 & $-15: 29: 28.5$ & $18.929(0.056)$ & $17.926(0.098)$ & \\
\hline 306 & 00:01 & $-15: 27: 37.4$ & $18.643(0.047)$ & $17.283(0.083)$ & BD04 \\
\hline 307 & 00:0 & $-15: 26: 39.1$ & $19.080(0.089)$ & $18.276(0.171)$ & \\
\hline 308 & 00: & -15 & 18. & 17. & BD \\
\hline 309 & 00: & -15 & 18. & 16. & $\mathrm{C}$ \\
\hline 310 & $00: 0$ & -15 & 18.6 & 17.8 & \\
\hline 311 & 00:0 & $-15: 25: 43.2$ & 18.8 & 17.938 & \\
\hline 312 & 00:01:59.01 & $-15: 26: 31.4$ & $19.080(0.063)$ & $18.245(0.129)$ & \\
\hline 313 & 00:01:59.02 & $-15: 28: 21.4$ & $18.344(0.037)$ & $16.709(0.031)$ & BD04 \\
\hline 314 & 00:01:59.02 & $-15: 28: 47.1$ & $19.566(0.082)$ & $18.824(0.223)$ & \\
\hline 315 & 00:01:59.06 & $-15: 28: 32.2$ & $19.542(0.106)$ & $17.798(0.092)$ & $\mathrm{C}$ \\
\hline 316 & 00:01:59.07 & $-15: 27: 18.6$ & $18.634(0.061)$ & $18.169(0.140)$ & \\
\hline 317 & 00:01:59.09 & $-15: 27: 00.9$ & $18.177(0.035)$ & $16.324(0.024)$ & $\mathrm{BD}$ \\
\hline 318 & 00:01:59.09 & $-15: 30: 04.0$ & $18.872(0.061)$ & $17.228(0.054)$ & BD04 \\
\hline 319 & 00:01:59.15 & $-15: 28: 44.8$ & $19.502(0.084)$ & $18.688(0.194)$ & \\
\hline 320 & 00:01:59.16 & $-15: 27: 15.0$ & $19.608(0.098)$ & $19.265(0.366)$ & \\
\hline 321 & 00:01:59.16 & $-15: 28: 09.5$ & $19.157(0.084)$ & $18.061(0.108)$ & \\
\hline 322 & 00:01:59.19 & $-15: 29: 55.4$ & $19.771(0.105)$ & $18.260(0.141)$ & $C$ \\
\hline 323 & 00:01:59.20 & $-15: 28: 12.9$ & $18.236(0.051)$ & $16.896(0.047)$ & BD04 \\
\hline 324 & 00:01:59.20 & $-15: 28: 33.5$ & $18.479(0.037)$ & $17.749(0.078)$ & \\
\hline 325 & 00:01:59.21 & $-15: 25: 52.4$ & $19.840(0.146)$ & $19.027(0.266)$ & \\
\hline 326 & 00:01:59.23 & $-15: 25: 20.5$ & $19.904(0.147)$ & $18.937(0.294)$ & \\
\hline
\end{tabular}


Table 1. continued.

\begin{tabular}{|c|c|c|c|c|c|}
\hline No & $\overline{\mathrm{RA}}$ & $\overline{\mathrm{Dec}}$ & $\bar{J}$ & $\overline{\overline{K_{\mathrm{S}}}}$ & Note \\
\hline 327 & $00: 01: 59.24$ & $-15: 29: 25.3$ & $19.131(0.081)$ & $17.754(0.090)$ & BD04 \\
\hline 328 & 00:01:59.28 & $-15: 28: 30.9$ & $18.885(0.060)$ & $18.451(0.152)$ & \\
\hline 329 & 00:01:59.30 & $-15: 25: 50.2$ & $18.713(0.052)$ & $17.274(0.053)$ & $\mathrm{C}$ \\
\hline 330 & 00:01:59.31 & $-15: 26: 07.0$ & $20.066(0.184)$ & $18.903(0.239)$ & \\
\hline 331 & 00:01:59.35 & $-15: 26: 45.8$ & $19.930(0.117)$ & $19.173(0.320)$ & \\
\hline 332 & 00:01:59.36 & $-15: 27: 00.8$ & $18.983(0.063)$ & $18.332(0.142)$ & \\
\hline 333 & 00:01:59.38 & $-15: 25: 22.4$ & $18.769(0.061)$ & $17.738(0.116)$ & \\
\hline 334 & 00:01:59.38 & $-15: 26: 41.4$ & $20.286(0.204)$ & $19.029(0.276)$ & \\
\hline 335 & 00:01:59.38 & $-15: 27: 47.7$ & $18.898(0.057)$ & $18.015(0.115)$ & \\
\hline 336 & 00:01:59.39 & $-15: 25: 36.6$ & $19.313(0.091)$ & $16.595(0.032)$ & $\mathrm{C}$ \\
\hline 337 & 00:01:59.39 & $-15: 26: 24.2$ & $19.155(0.077)$ & $18.381(0.159)$ & \\
\hline 338 & 00:01:59.40 & $-15: 25: 47.3$ & $16.527(0.019)$ & $15.713(0.015)$ & \\
\hline 339 & 00:01:59.40 & $-15: 28: 21.6$ & $19.913(0.144)$ & $19.399(0.453)$ & \\
\hline 340 & 00:01:59.40 & $-15: 29: 14.3$ & $18.366(0.041)$ & $17.593(0.071)$ & \\
\hline 341 & 00:01:59.40 & $-15: 29: 44.7$ & $20.381(0.201)$ & $19.380(0.392)$ & \\
\hline 342 & 00:01:59.43 & $-15: 25: 50.7$ & $20.201(0.215)$ & $19.034(0.263)$ & BD04 \\
\hline 343 & 00:01:59.43 & $-15: 27: 37.6$ & $18.274(0.043)$ & $17.256(0.057)$ & \\
\hline 344 & 00:01:59.44 & $-15: 25: 44.0$ & $19.215(0.074)$ & $18.260(0.138)$ & \\
\hline 345 & 00:01:59.44 & $-15: 26: 20.7$ & $19.479(0.094)$ & $18.489(0.172)$ & \\
\hline 346 & 00:01:59.46 & $-15: 29: 40.4$ & $19.978(0.161)$ & $18.913(0.255)$ & \\
\hline 347 & 00:01:59.47 & $-15: 27: 04.1$ & $20.234(0.197)$ & $19.295(0.363)$ & \\
\hline 348 & 00:01:59.48 & $-15: 25: 34.2$ & $19.398(0.104)$ & $18.604(0.188)$ & \\
\hline 349 & 00:01:59.49 & $-15: 27: 56.6$ & $17.894(0.040)$ & $17.129(0.047)$ & \\
\hline 350 & 00:01:59.50 & $-15: 27: 34.9$ & $19.069(0.077)$ & $18.336(0.142)$ & \\
\hline 351 & 00:01:59.50 & $-15: 29: 27.4$ & $17.930(0.029)$ & $17.812(0.080)$ & \\
\hline 352 & 00:01:59.52 & $-15: 27: 51.2$ & $19.401(0.082)$ & $18.965(0.254)$ & \\
\hline 353 & 00:01:59.55 & $-15: 25: 50.6$ & $19.829(0.152)$ & $19.036(0.247)$ & \\
\hline 354 & 00:01:59.56 & $-15: 29: 49.0$ & $20.042(0.447)$ & $16.163(0.025)$ & $\mathrm{C}$ \\
\hline 355 & 00:01:59.62 & $-15: 29: 50.9$ & $19.316(0.082)$ & $18.392(0.140)$ & \\
\hline 356 & 00:01:59.64 & $-15: 28: 33.8$ & $19.678(0.102)$ & $18.281(0.144)$ & $\mathrm{C}$ \\
\hline 357 & 00:01:59.66 & $-15: 26: 48.7$ & $19.817(0.136)$ & $18.901(0.238)$ & \\
\hline 358 & 00:01:59.68 & $-15: 27: 29.5$ & $18.321(0.032)$ & 17.620 & \\
\hline 359 & 00:01:59.73 & $-15: 25: 43.6$ & $19.151(0.076)$ & 18.483 & \\
\hline 360 & 00:01:59.74 & $-15: 29: 42.5$ & $19.007(0.064)$ & $17.645(0.081)$ & BD04 \\
\hline 361 & 00:01:59.83 & $-15: 25: 22.2$ & $20.062(0.159)$ & $19.265(0.408)$ & \\
\hline 362 & 00:01:59.84 & $-15: 26: 42.2$ & $20.048(0.172)$ & $19.440(0.377)$ & \\
\hline 363 & 00:01:59.85 & $-15: 27: 18.8$ & $18.696(0.053)$ & $17.395(0.061)$ & BD04 \\
\hline 364 & 00:01:59.85 & $-15: 28: 20.0$ & $18.748(0.052)$ & $18.749(0.202)$ & \\
\hline 365 & 00:01:59.89 & $-15: 28: 30.3$ & $19.367(0.082)$ & $17.712(0.087)$ & $\mathrm{C}$ \\
\hline 366 & 00:01:59.90 & $-15: 26: 05.3$ & $18.662(0.054)$ & $17.537(0.068)$ & BD04 \\
\hline 367 & 00:01:59.90 & $-15: 29: 42.3$ & $18.831(0.055)$ & $16.724(0.038)$ & BD04 \\
\hline 368 & 00:01:59.91 & $-15: 27: 59.9$ & $17.951(0.032)$ & $17.073(0.048)$ & \\
\hline 369 & 00:01:59.93 & $-15: 25: 28.8$ & $18.112(0.028)$ & $16.678(0.035)$ & BD04 \\
\hline 370 & 00:01 & $-15: 27: 31.7$ & $18.910(0.077)$ & $17.871(0.103)$ & \\
\hline 371 & 00:01 & $-15: 28: 32.0$ & $18.407(0.039)$ & $17.773(0.087)$ & \\
\hline 372 & 00:0 & $-15: 26$ & 19.0 & 18.307 & \\
\hline 373 & 00:01:59.98 & $-15: 29: 20.2$ & $19.076(0.064)$ & $17.672(0.084)$ & BD04 \\
\hline 374 & 00:02:00.00 & $-15: 26: 19.1$ & $18.086(0.032)$ & $17.097(0.048)$ & \\
\hline 375 & 00:02:00.00 & $-15: 28: 27.1$ & $18.940(0.065)$ & $18.067(0.116)$ & \\
\hline 376 & 00:02:00.01 & $-15: 26: 37.5$ & $18.796(0.057)$ & $17.489(0.066)$ & BD04 \\
\hline 377 & 00:02:00.02 & $-15: 25: 31.2$ & $18.373(0.042)$ & $17.405(0.066)$ & \\
\hline 378 & 00:02:00.03 & $-15: 25: 41.1$ & $20.214(0.166)$ & $18.959(0.262)$ & \\
\hline 379 & 00:02:00.04 & $-15: 28: 00.8$ & $19.886(0.129)$ & $19.024(0.274)$ & \\
\hline 380 & 00:02:00.04 & $-15: 28: 53.0$ & $20.074(0.141)$ & $18.296(0.145)$ & $\mathrm{C}$ \\
\hline 381 & 00:02:00.06 & $-15: 27: 41.1$ & $19.476(0.087)$ & $18.564(0.184)$ & \\
\hline 382 & 00:02:00.06 & $-15: 29: 53.7$ & $18.959(0.067)$ & $18.340(0.142)$ & \\
\hline 383 & 00:02:00.07 & $-15: 28: 14.3$ & $18.985(0.065)$ & $18.003(0.103)$ & \\
\hline 384 & 00:02:00.08 & $-15: 28: 34.0$ & $18.742(0.056)$ & $17.885(0.093)$ & \\
\hline 385 & 00:02:00.12 & $-15: 28: 02.1$ & $18.649(0.044)$ & $17.632(0.076)$ & \\
\hline 386 & 00:02:00.13 & $-15: 29: 28.8$ & $19.088(0.071)$ & $18.307(0.148)$ & \\
\hline 387 & 00:02:00.14 & $-15: 29: 11.0$ & $19.027(0.067)$ & $18.049(0.102)$ & \\
\hline 388 & 00:02:00.15 & $-15: 25: 26.9$ & $19.962(0.155)$ & $18.558(0.195)$ & $\mathrm{C}$ \\
\hline 389 & 00:02:00.22 & $-15: 28: 06.9$ & $19.748(0.102)$ & $19.011(0.258)$ & \\
\hline 390 & 00:02:00.25 & $-15: 28: 23.3$ & $18.811(0.053)$ & $17.776(0.092)$ & \\
\hline 391 & 00:02:00.26 & $-15: 29: 49.3$ & $18.288(0.058)$ & $16.583(0.059)$ & $\mathrm{C}$ \\
\hline 392 & 00:02:00.27 & $-15: 27: 02.7$ & $18.818(0.049)$ & $17.729(0.091)$ & \\
\hline
\end{tabular}


Table 1. continued.

\begin{tabular}{|c|c|c|c|c|c|}
\hline No & RA & Dec & $J$ & $K_{\mathrm{S}}$ & Note \\
\hline 393 & $00: 02: 00.27$ & $-15: 28: 47.0$ & $20.043(0.137)$ & $19.039(0.285)$ & \\
\hline 394 & 00:02:00.28 & $-15: 25: 56.0$ & $20.287(0.194)$ & $19.484(0.456)$ & \\
\hline 395 & 00:02:00.30 & $-15: 25: 35.3$ & $18.786(0.049)$ & $17.746(0.086)$ & \\
\hline 396 & 00:02:00.31 & $-15: 27: 38.5$ & $19.245(0.085)$ & $18.506(0.159)$ & \\
\hline 397 & $00: 02: 00.33$ & $-15: 26: 38.3$ & $19.047(0.064)$ & $18.487(0.161)$ & \\
\hline 398 & 00:02:00.35 & $-15: 27: 03.5$ & $18.544(0.077)$ & $16.917(0.070)$ & $\mathrm{C}$ \\
\hline 399 & 00:02:00.37 & $-15: 28: 51.2$ & $19.357(0.074)$ & $18.503(0.155)$ & \\
\hline 400 & $00: 02: 00.41$ & $-15: 28: 20.7$ & $19.606(0.108)$ & $18.532(0.175)$ & \\
\hline 401 & 00:02:00.42 & $-15: 29: 59.2$ & $18.383(0.045)$ & $17.071(0.045)$ & BD04 \\
\hline 402 & 00:02:00.43 & $-15: 28: 18.5$ & $19.302(0.079)$ & $18.350(0.131)$ & \\
\hline 403 & 00:02:00.44 & $-15: 26: 48.1$ & $19.448(0.085)$ & $18.531(0.177)$ & \\
\hline 404 & 00:02:00.44 & $-15: 27: 22.9$ & $19.024(0.063)$ & $18.402(0.174)$ & \\
\hline 405 & 00:02:00.46 & $-15: 28: 51.4$ & $19.437(0.082)$ & $18.519(0.166)$ & \\
\hline 406 & 00:02:00.46 & $-15: 29: 00.9$ & $18.606(0.053)$ & $17.518(0.073)$ & \\
\hline 407 & 00:02:00.52 & $-15: 27: 33.6$ & $18.784(0.054)$ & $17.645(0.077)$ & \\
\hline 408 & $00: 02: 00.53$ & $-15: 28: 31.0$ & $18.161(0.033)$ & $17.937(0.122)$ & \\
\hline 409 & 00:02:00.57 & $-15: 28: 02.2$ & $18.765(0.051)$ & $18.041(0.104)$ & \\
\hline 410 & 00:02:00.57 & $-15: 29: 03.9$ & $18.621(0.054)$ & $18.060(0.110)$ & \\
\hline 411 & 00:02:00.59 & $-15: 29: 29.8$ & $18.794(0.055)$ & $17.453(0.068)$ & BD04 \\
\hline 412 & 00:02:00.64 & $-15: 26: 59.8$ & $18.997(0.060)$ & $17.943(0.091)$ & \\
\hline 413 & 00:02:00.65 & $-15: 26: 18.2$ & $19.072(0.085)$ & $18.665(0.216)$ & \\
\hline 414 & $00: 02: 00.65$ & $-15: 27: 25.3$ & $18.806(0.052)$ & $17.323(0.056)$ & $\mathrm{C}$ \\
\hline 415 & 00:02:00.71 & $-15: 26: 22.7$ & $19.309(0.086)$ & $18.474(0.167)$ & SC85 V28 \\
\hline 416 & 00:02:00.74 & $-15: 25: 36.9$ & $20.631(0.270)$ & $19.371(0.380)$ & \\
\hline 417 & 00:02:00.74 & $-15: 26: 30.1$ & $18.391(0.042)$ & $17.479(0.065)$ & \\
\hline 418 & 00:02:00.75 & $-15: 25: 26.9$ & $19.888(0.151)$ & $18.721(0.242)$ & \\
\hline 419 & 00:02:00.77 & $-15: 27: 23.1$ & $20.518(0.232)$ & $19.695(0.530)$ & BD04 \\
\hline 420 & 00:02:00.88 & $-15: 28: 16.5$ & $19.029(0.062)$ & $17.639(0.074)$ & $\mathrm{C}$ \\
\hline 421 & 00:02:00.89 & $-15: 28: 47.2$ & $19.265(0.072)$ & $18.236(0.135)$ & \\
\hline 422 & 00:02:00.95 & $-15: 27: 47.7$ & $19.555(0.094)$ & $18.659(0.192)$ & \\
\hline 423 & 00:02:00.99 & $-15: 27: 00.3$ & $19.241(0.076)$ & $18.116(0.118)$ & \\
\hline 424 & 00:02:01.00 & $-15: 28: 02.8$ & $20.116(0.166)$ & $19.009(0.262)$ & \\
\hline 425 & 00:02:01.00 & $-15: 28: 36.1$ & $16.843(0.025)$ & $15.953(0.019)$ & SC85 V32 LC \\
\hline 426 & 00:02:01.02 & $-15: 25: 27.8$ & $20.293(0.201)$ & $19.319(0.406)$ & \\
\hline 427 & 00:02:01.02 & $-15: 25: 44.8$ & $18.601(0.043)$ & $17.327(0.064)$ & $\mathrm{C}$ \\
\hline 428 & 00:02:01.06 & $-15: 25: 32.0$ & $20.172(0.197)$ & $19.210(0.357)$ & \\
\hline 429 & 00:02:01.06 & $-15: 29: 56.9$ & $18.566(0.049)$ & $16.930(0.045)$ & BD04 \\
\hline 430 & 00:02:01.07 & $-15: 26: 22.8$ & $19.265(0.077)$ & $18.360(0.153)$ & \\
\hline 431 & 00:02:01.09 & $-15: 27: 23.1$ & $20.208(0.162)$ & $19.123(0.292)$ & \\
\hline 432 & 00:02:01.11 & $-15: 25: 43.1$ & $19.745(0.118)$ & $18.637(0.204)$ & BD04 \\
\hline 433 & 00:02:01.14 & $-15: 30: 07.8$ & $19.494(0.091)$ & $18.668(0.220)$ & \\
\hline 434 & 00:02:01.16 & $-15: 27: 42.3$ & $19.179(0.075)$ & $18.270(0.130)$ & \\
\hline 435 & 00:02:01.17 & $-15: 25: 31.3$ & $19.795(0.132)$ & $18.689(0.218)$ & \\
\hline 436 & 00:02:01.20 & $-15: 29: 13.8$ & $20.154(0.151)$ & $18.977(0.262)$ & \\
\hline 437 & 00:02:01.22 & $-15: 27: 15.3$ & $18.271(0.041)$ & $16.845(0.036)$ & BD04 \\
\hline 438 & 00:02:01.26 & $-15: 25: 38.8$ & $19.218(0.066)$ & $17.304(0.061)$ & BD04 \\
\hline 439 & 00:02:01.29 & $-15: 27: 29.2$ & $19.117(0.079)$ & $18.028(0.102)$ & \\
\hline 440 & 00:02:01.31 & $-15: 29: 17.2$ & $19.505(0.084)$ & $16.721(0.040)$ & $\mathrm{C}$ \\
\hline 441 & 00:02:01.32 & $-15: 26: 15.0$ & $19.345(0.083)$ & $18.193(0.125)$ & \\
\hline 442 & 00:02:01.32 & $-15: 27: 41.8$ & $18.479(0.043)$ & $17.509(0.071)$ & \\
\hline 443 & 00:02:01.33 & $-15: 27: 00.2$ & $19.356(0.089)$ & $18.181(0.123)$ & \\
\hline 444 & 00:02:01.33 & $-15: 28: 38.2$ & $17.521(0.029)$ & $16.078(0.022)$ & $\mathrm{C}$ \\
\hline 445 & 00:02:01.34 & $-15: 27: 31.1$ & $17.171(0.026)$ & $16.777(0.036)$ & \\
\hline 446 & 00:02:01.34 & $-15: 29: 20.6$ & $19.205(0.083)$ & $17.679(0.090)$ & BD04 \\
\hline 447 & 00:02:01.38 & $-15: 28: 47.3$ & $20.023(0.147)$ & $18.618(0.196)$ & $\mathrm{C}$ \\
\hline 448 & 00:02:01.40 & $-15: 27: 28.9$ & $18.544(0.048)$ & $16.866(0.038)$ & BD04 \\
\hline 449 & 00:02:01.41 & $-15: 26: 47.4$ & $18.530(0.045)$ & $17.855(0.099)$ & \\
\hline 450 & 00:02:01.43 & $-15: 27: 50.2$ & $19.919(0.154)$ & $19.306(0.360)$ & \\
\hline 451 & 00:02:01.45 & $-15: 25: 19.7$ & $20.056(0.216)$ & $19.147(0.456)$ & \\
\hline 452 & 00:02:01.45 & $-15: 28: 28.1$ & $19.186(0.079)$ & $18.224(0.130)$ & \\
\hline
\end{tabular}


Table 1. continued.

\begin{tabular}{|c|c|c|c|c|c|}
\hline$\overline{\mathrm{No}}$ & $\overline{\overline{\mathrm{RA}}}$ & $\overline{\overline{\text { Dec }}}$ & $\overline{\bar{J}}$ & $\overline{\overline{K_{\mathrm{S}}}}$ & $\overline{\text { Note }}$ \\
\hline 453 & $00: 02: 01.48$ & $-15: 26: 05.3$ & $19.326(0.087)$ & $18.242(0.122)$ & \\
\hline 454 & 00:02:01.50 & $-15: 27: 14.2$ & $20.488(0.231)$ & $17.020(0.043)$ & $\mathrm{C}$ \\
\hline 455 & 00:02:01.50 & $-15: 28: 38.1$ & $19.008(0.057)$ & $18.031(0.103)$ & BD04 \\
\hline 456 & 00:02:01.54 & $-15: 26: 52.5$ & $17.891(0.086)$ & $16.455(0.052)$ & $\mathrm{C}$ \\
\hline 457 & 00:02:01.58 & $-15: 26: 52.4$ & $18.704(0.168)$ & $16.455(0.052)$ & $\mathrm{C}$ \\
\hline 458 & 00:02:01.62 & $-15: 29: 27.5$ & $19.539(0.088)$ & $17.988(0.108)$ & BD04 \\
\hline 459 & 00:02:01.66 & $-15: 25: 49.9$ & $19.032(0.058)$ & $18.187(0.126)$ & \\
\hline 460 & 00:02:01.73 & $-15: 26: 11.3$ & $16.879(0.024)$ & $15.839(0.016)$ & \\
\hline 461 & 00:02:01.74 & $-15: 28: 45.7$ & $18.914(0.062)$ & $17.511(0.069)$ & $\mathrm{C}$ \\
\hline 462 & 00:02:01.76 & $-15: 29: 07.9$ & $19.467(0.099)$ & $18.282(0.140)$ & \\
\hline 463 & 00:02:01.81 & $-15: 28: 52.5$ & $20.263(0.174)$ & $19.095(0.301)$ & \\
\hline 464 & 00:02:01.82 & $-15: 29: 36.7$ & $18.932(0.056)$ & $17.630(0.082)$ & $\mathrm{C}$ \\
\hline 465 & 00:02:01.89 & $-15: 25: 49.9$ & $19.988(0.154)$ & $18.885(0.259)$ & \\
\hline 466 & 00:02:01.89 & $-15: 26: 23.0$ & $16.106(0.020)$ & $15.371(0.012)$ & \\
\hline 467 & 00:02:01.94 & $-15: 27: 47.9$ & $18.434(0.045)$ & $17.035(0.049)$ & BD04 \\
\hline 468 & 00:02:01.96 & $-15: 27: 02.7$ & $19.869(0.140)$ & $17.260(0.057)$ & $\mathrm{C}$ \\
\hline 469 & 00:02:01.99 & $-15: 28: 30.1$ & $19.267(0.082)$ & $17.809(0.096)$ & BD04 \\
\hline 470 & 00:02:02.01 & $-15: 25: 25.3$ & $19.233(0.078)$ & $18.033(0.128)$ & \\
\hline 471 & 00:02:02.04 & $-15: 26: 04.1$ & $19.317(0.081)$ & $18.211(0.124)$ & \\
\hline 472 & 00:02:02.04 & $-15: 29: 03.0$ & $19.934(0.131)$ & $18.651(0.190)$ & $\mathrm{C}$ \\
\hline 473 & 00:02:02.05 & $-15: 26: 17.0$ & $19.917(0.147)$ & $18.903(0.264)$ & \\
\hline 474 & 00:02:02.05 & $-15: 26: 20.5$ & $19.256(0.107)$ & $18.208(0.138)$ & \\
\hline 475 & 00:02:02.05 & $-15: 26: 22.3$ & $18.699(0.055)$ & $16.831(0.033)$ & $\mathrm{C}$ \\
\hline 476 & 00:02:02.10 & $-15: 27: 33.7$ & $20.190(0.167)$ & $19.118(0.327)$ & \\
\hline 477 & 00:02:02.11 & $-15: 28: 51.2$ & $20.014(0.140)$ & $18.952(0.280)$ & \\
\hline 478 & 00:02:02.12 & $-15: 26: 55.4$ & $19.930(0.155)$ & $18.814(0.223)$ & \\
\hline 479 & 00:02:02.16 & $-15: 26: 19.9$ & $17.767(0.031)$ & $16.644(0.034)$ & BD04 \\
\hline 480 & 00:02:02.17 & $-15: 28: 33.7$ & $19.433(0.140)$ & $18.237(0.152)$ & \\
\hline 481 & 00:02:02.17 & $-15: 29: 37.1$ & $19.356(0.075)$ & $18.343(0.137)$ & \\
\hline 482 & 00:02:02.20 & $-15: 27: 44.3$ & $18.639(0.052)$ & $17.291(0.058)$ & $\mathrm{C}$ \\
\hline 483 & 00:02:02.23 & $-15: 27: 35.7$ & $19.561(0.103)$ & $18.520(0.176)$ & \\
\hline 484 & 00:02:02.25 & $-15: 26: 01.4$ & $18.924(0.059)$ & $17.942(0.096)$ & \\
\hline 485 & $00: 02: 02.25$ & $-15: 27: 29.3$ & $19.720(0.110)$ & $19.334(0.378)$ & \\
\hline 486 & 00:02:02.27 & $-15: 26: 53.7$ & $19.850(0.127)$ & $18.975(0.261)$ & \\
\hline 487 & 00:02:02.33 & $-15: 25: 51.0$ & $19.062(0.071)$ & $17.315(0.056)$ & BD04 \\
\hline 488 & 00:02:02.35 & $-15: 26: 24.6$ & $19.473(0.094)$ & $18.742(0.223)$ & \\
\hline 489 & 00:02:02.38 & $-15: 26: 10.8$ & $19.493(0.096)$ & $17.946(0.115)$ & BD04 \\
\hline 490 & 00:02:02.39 & $-15: 26: 33.3$ & $19.782(0.117)$ & $19.081(0.288)$ & \\
\hline 491 & 00:02:02.40 & $-15: 28: 12.2$ & $18.817(0.063)$ & $17.086(0.051)$ & BD04 \\
\hline 492 & 00:02:02.43 & $-15: 27: 16.8$ & $19.745(0.139)$ & $18.814(0.228)$ & \\
\hline 493 & 00:02:02.52 & $-15: 27: 32.1$ & $20.331(0.192)$ & $19.514(0.481)$ & \\
\hline 494 & 00:02:02.60 & $-15: 28: 21.8$ & $20.160(0.167)$ & $18.931(0.245)$ & \\
\hline 495 & 00:02:02.60 & $-15: 29: 49.3$ & $20.138(0.159)$ & $19.184(0.305)$ & \\
\hline 496 & 00:02:02.61 & $-15: 27: 49.8$ & $19.735(0.109)$ & $18.827(0.248)$ & \\
\hline 497 & 00:02:02.67 & $-15: 26: 03.3$ & $19.213(0.077)$ & $18.165(0.132)$ & \\
\hline 498 & 00:02:02.68 & $-15: 26: 24.1$ & $20.889(0.372)$ & $19.108(0.288)$ & \\
\hline 499 & 00:02:02.68 & $-15: 29: 14.8$ & $19.019(0.058)$ & $17.928(0.103)$ & \\
\hline 500 & 00:02:02.69 & $-15: 29: 01.2$ & $18.989(0.056)$ & $17.001(0.048)$ & $\mathrm{C}$ \\
\hline 501 & 00:02:02.71 & $-15: 28: 14.5$ & $20.125(0.146)$ & $19.279(0.356)$ & \\
\hline 502 & 00:02:02.74 & $-15: 27: 16.8$ & $18.478(0.062)$ & $16.787(0.067)$ & $\mathrm{C}$ \\
\hline 503 & 00:02:02.77 & $-15: 27: 55.0$ & $19.327(0.072)$ & $16.825(0.040)$ & $\mathrm{C}$ \\
\hline 504 & 00:02:02.83 & $-15: 29: 58.7$ & $18.806(0.052)$ & $17.046(0.056)$ & $\mathrm{C}$ \\
\hline 505 & 00:02:02.84 & $-15: 26: 38.2$ & $19.401(0.082)$ & $18.424(0.171)$ & \\
\hline 506 & 00:02:03.04 & $-15: 28: 24.1$ & $18.743(0.051)$ & $16.997(0.041)$ & $\mathrm{C}$ \\
\hline 507 & 00:02:03.07 & $-15: 28: 17.0$ & $18.662(0.042)$ & $18.239(0.143)$ & \\
\hline 508 & 00:02:03.09 & $-15: 26: 17.5$ & $18.813(0.056)$ & $17.249(0.056)$ & $\mathrm{C}$ \\
\hline 509 & 00:02:03.10 & $-15: 28: 10.9$ & $19.643(0.116)$ & $18.693(0.195)$ & \\
\hline 510 & 00:02:03.14 & $-15: 27: 56.0$ & $19.806(0.134)$ & $19.117(0.339)$ & \\
\hline 511 & 00:02:03.18 & $-15: 25: 39.1$ & $17.786(0.026)$ & $16.621(0.033)$ & \\
\hline 512 & 00:02:03.28 & $-15: 28: 57.5$ & $20.162(0.155)$ & $19.096(0.296)$ & \\
\hline 513 & 00:02:03.34 & $-15: 25: 47.6$ & $19.513(0.107)$ & $18.286(0.150)$ & $\mathrm{C}$ \\
\hline 514 & 00:02:03.43 & $-15: 26: 22.8$ & $17.998(0.032)$ & $17.161(0.052)$ & SC85 V \\
\hline 515 & 00:02:03.46 & $-15: 29: 50.0$ & $19.966(0.144)$ & $18.290(0.141)$ & $\mathrm{C}$ \\
\hline 516 & 00:02:03.49 & $-15: 25: 48.2$ & $20.347(0.187)$ & $19.165(0.326)$ & \\
\hline
\end{tabular}


A. T. Valcheva et al.: Near-IR view of WLM: carbon stars and C/M ratio, Online Material p 10

Table 1. continued.

\begin{tabular}{|c|c|c|c|c|c|}
\hline No & $\overline{\mathrm{RA}}$ & $\overline{\mathrm{Dec}}$ & $\bar{J}$ & $\overline{K_{\mathrm{S}}}$ & Note \\
\hline 517 & $00: 02: 03.50$ & $-15: 26: 10.1$ & $20.057(0.182)$ & $18.957(0.283)$ & \\
\hline 518 & 00:02:03.56 & $-15: 27: 23.9$ & $18.755(0.059)$ & $17.132(0.068)$ & $\mathrm{C}$ \\
\hline 519 & 00:02:03.58 & $-15: 26: 18.0$ & $19.208(0.079)$ & $18.096(0.132)$ & \\
\hline 520 & 00:02:03.61 & $-15: 27: 13.6$ & 20.087 (0.181) & $19.413(0.473)$ & \\
\hline 521 & 00:02:03.62 & $-15: 26: 10.9$ & $20.674(0.294)$ & $19.478(0.458)$ & \\
\hline 522 & $00: 02: 03.62$ & $-15: 27: 06.0$ & $20.180(0.190)$ & $19.002(0.299)$ & \\
\hline 523 & 00:02:03.64 & $-15: 29: 57.5$ & $20.524(0.255)$ & $19.022(0.325)$ & \\
\hline 524 & 00:02:03.65 & $-15: 27: 02.1$ & $19.242(0.087)$ & $18.216(0.175)$ & \\
\hline 525 & 00:02:03.66 & $-15: 27: 00.0$ & $18.157(0.046)$ & $17.179(0.059)$ & \\
\hline 526 & 00:02:03.71 & $-15: 25: 48.1$ & $19.933(0.165)$ & $18.688(0.238)$ & $\mathrm{C}$ \\
\hline 527 & 00:02:03.73 & $-15: 25: 38.4$ & $19.984(0.134)$ & $19.031(0.366)$ & \\
\hline 528 & 00:02:03.73 & $-15: 27: 07.6$ & $19.076(0.071)$ & $17.405(0.074)$ & $\mathrm{C}$ \\
\hline 529 & 00:02:03.75 & $-15: 27: 33.6$ & $19.332(0.092)$ & $18.308(0.170)$ & \\
\hline 530 & 00:02:03.76 & $-15: 25: 43.5$ & $18.961(0.068)$ & $17.706(0.116)$ & $\mathrm{C}$ \\
\hline 531 & 00:02:03.77 & $-15: 26: 08.7$ & $20.206(0.200)$ & $19.226(0.431)$ & \\
\hline 532 & 00:02:03.78 & $-15: 28: 47.6$ & $19.561(0.124)$ & $17.857(0.129)$ & $\mathrm{C}$ \\
\hline 533 & 00:02:03.80 & $-15: 26: 25.8$ & $20.254(0.198)$ & $19.299(0.438)$ & \\
\hline 534 & 00:02:03.83 & $-15: 26: 17.2$ & $20.352(0.229)$ & $19.286(0.512)$ & \\
\hline 535 & 00:02:03.84 & $-15: 27: 48.9$ & $20.154(0.190)$ & $17.218(0.069)$ & $\mathrm{C}$ \\
\hline 536 & 00:02:03.85 & $-15: 25: 39.2$ & $20.323(0.244)$ & $19.453(0.514)$ & \\
\hline 537 & 00:02:03.85 & $-15: 28: 13.7$ & $20.634(0.306)$ & $19.454(0.528)$ & \\
\hline 538 & 00:02:03.90 & $-15: 25: 47.9$ & $15.330(0.042)$ & $14.538(0.014)$ & \\
\hline 539 & 00:02:03.92 & $-15: 29: 30.3$ & $19.927(0.136)$ & $19.397(0.488)$ & \\
\hline 540 & 00:02:03.95 & $-15: 25: 40.6$ & $20.528(0.293)$ & $18.853(0.299)$ & \\
\hline 541 & 00:02:03.98 & $-15: 26: 30.2$ & $19.160(0.072)$ & $17.724(0.094)$ & $\mathrm{C}$ \\
\hline 542 & 00:02:04.03 & $-15: 28: 02.4$ & $19.726(0.145)$ & $18.585(0.238)$ & \\
\hline 543 & 00:02:04.07 & $-15: 27: 57.6$ & $19.509(0.102)$ & $19.254(0.413)$ & \\
\hline 544 & 00:02:04.10 & $-15: 29: 42.3$ & $19.175(0.086)$ & $18.862(0.315)$ & \\
\hline 545 & 00:02:04.14 & $-15: 28: 19.4$ & $20.080(0.199)$ & $19.128(0.369)$ & \\
\hline 546 & 00:02:04.17 & $-15: 28: 43.9$ & $21.066(0.421)$ & $19.635(0.516)$ & \\
\hline 547 & 00:02:04.24 & $-15: 28: 07.5$ & $19.461(0.108)$ & $17.947(0.136)$ & $\mathrm{C}$ \\
\hline 548 & 00:02:04.31 & $-15: 29: 17.9$ & $20.089(0.182)$ & $18.977(0.338)$ & \\
\hline 549 & 00:02:04.33 & $-15: 29: 11.2$ & $19.269(0.079)$ & $17.796(0.118)$ & $\mathrm{C}$ \\
\hline 550 & 00:02:04.42 & $-15: 25: 42.0$ & $20.075(0.177)$ & $18.779(0.355)$ & \\
\hline 551 & 00:02:04.46 & $-15: 26: 14.1$ & $20.598(0.299)$ & $18.971(0.375)$ & \\
\hline 552 & 00:02:04.47 & $-15: 27: 16.0$ & $19.690(0.129)$ & $17.135(0.088)$ & $\mathrm{C}$ \\
\hline 553 & 00:02:04.52 & $-15: 27: 44.5$ & $19.947(0.159)$ & $19.182(0.486)$ & \\
\hline 554 & 00:02:04.52 & $-15: 29: 37.6$ & $18.040(0.046)$ & $16.760(0.126)$ & $c$ \\
\hline 555 & 00:02:04.60 & $-15: 27: 07.5$ & $20.153(0.194)$ & $19.296(0.533)$ & \\
\hline
\end{tabular}

